J. Nonlinear Var. Anal. 4 (2020), No. 2, pp. 163-188

Available online at http://jnva.biemdas.com

https://doi.org/10.23952/jnva.4.2020.2.02

\title{
A STAND-ALONE ANALYSIS OF QUASIDENSITY
}

\author{
STEPHEN SIMONS \\ Department of Mathematics, University of California, Santa Barbara, CA 93106-3080, USA
}

This paper is dedicated to my good friend Juan Enrique Martínez-Legaz, with fond memories of an earthshaking moment that we spent together.

\begin{abstract}
In this paper, we consider the "quasidensity" of a subset of the product of a Banach space and its dual, and give a connection between quasidense sets and sets of "type (NI)". We discuss "coincidence sets" of certain convex functions and prove two sum theorems for coincidence sets. We obtain new results on the Fitzpatrick extension of a closed quasidense monotone multifunction. The analysis in this paper is self-contained, and independent of previous work on "Banach SN spaces".
\end{abstract}

Keywords. Fenchel conjugate; Maximal monotonicity, Quasidensity; Sum theorem; Subdifferential.

\section{INTRODUCTION}

In this paper, we suppose that $E$ is a nonzero real Banach space with dual $E^{*}$. In [19], we defined the quasidensity of a subset of $E \times E^{*}$. This was actually a special case of the concept of the $r_{L}$-density of a subset of a Banach SN space that had been previously defined in [18], and the analysis in [19] was heavily dependent on [18]. The purpose of this paper is to give a development of the properties of quasidensity that is independent of [18]. This paper also contains many results that did not appear in [19].

In Section 2, we discuss proper convex functions on a Banach space and their Fenchel conjugates and biconjugates. We also introduce the (well known) canonical map from a Banach space into its bidual, which we denote by $\widehat{~}$. In Theorems 2.1 and 2.3 and Lemma 2.2, we discuss some subtler properties of proper convex functions that are not necessarily lower semicontinuous. These subtler properties will be used in Theorem 4.11.

In Section 3, we discuss Banach spaces of the form $E \times E^{*}$. For this kind of Banach space, there is a (not so well known) canonical map from the space into its dual, which we denote by $L$ (see (3.1)). We define the quasidensity of of a subset of $E \times E^{*}$ (or, equivalently, of a multifunction from $E$ into $E^{*}$ ) in Definition 3.1. The definition of quasidensity does not require monotonicity, though there is a rich theory of the interaction of quasidensity and monotonicity which we will discuss in Sections 6-12 the definition of monotonicity does not actually appear until Section 6. Lemma 3.3, Theorem 3.5 and Corollaries 3.7 and 3.9 contain useful results on quasidensity without a monotonicity assumption. In particular, Theorem 3.5 says that $L$ "preserves quasidensity", and we establish in Corollary 3.7 that every quasidense set is of type (NI), a concept that has been extensively studied over the past two decades. We will return to this issue below. We mention in Example 3.2 that the subdifferential of a proper, convex,

E-mail address: stesim38@gmail.com.

Received October 28, 2019; Accepted May 3, 2020.

(C)2020 Journal of Nonlinear and Variational Analysis 
lower semicontinuous function on $E$ is quasidense. This result is generalized in [20] to certain more general subdifferentials of nonconvex functions.

In Section 4, we initiate the theory of the coincidence sets of certain convex functions. The basic idea is that we consider a proper convex function, $f$, on $E \times E^{*}$ that dominates the canonical bilinear form, $q_{L}$, and the corresponding coincidence set is the set on which $f$ and $q_{L}$ coincide. (The " $q$ " in this notation stands for "quadratic".) The main results in this section (and the pivotal results of this paper) are Theorem 4.4 (the primal condition for quasidensity), Theorem 4.8 (the dual condition for quasidensity) and Theorem 4.11 (the theorem of the three functions). As we observed above, the definition of monotonicity is not used explicitly before Section 6, but monotonicity is hiding below the surface because, as we shall see in Lemma 6.2, coincidence sets are monotone.

In Section 5, we investigate the coincidence sets of the partial episums of a pair of convex functions. This analysis will lead to the two sum theorems for quasidense maximally monotone multifunctions that we will establish in Theorem 7.3 and Theorem 9.1.

We start our explicit discussion of monotonicity in Section 6. We prove in Theorem 6.1 that every closed, monotone quasidense multifunction is maximally monotone. On the other hand, we give examples of varying degrees of abstraction in Example 8.8 and Theorems 10.3, 11.4(b) and 12.3 of maximally monotone linear operators that are not quasidense. The link between Section 4 and Section 6 is provided by Lemma 6.2, in which we give a short proof of the result first established by Burachik-Svaiter and Penot that coincidence sets are monotone. So suppose that $S: E \rightrightarrows E^{*}$ is monotone and $G(S) \neq \emptyset$. In Definition 6.3, we define the function, $\left.\left.\theta_{S}: E^{*} \times E^{* *} \rightarrow\right]-\infty, \infty\right]$, by adapting Definition 3.8. The well known Fitzpatrick function, $\varphi_{S}$, is defined in Definition 6.4 by $\varphi_{S}=\theta_{S} \circ L$. There is a short history of the Fitzpatrick function in Remark 6.5. Now let $S$ be maximally monotone. Then we prove in Theorem 6.7 that $S$ is quasidense if, and only if, $\varphi_{S}{ }^{*} \geq q_{\widetilde{L}}$ on $E^{*} \times E^{* *}$, and we prove in Theorem 6.12 that $S$ is quasidense if, and only if, $\theta_{S} \geq q_{\widetilde{L}}$ on $E^{*} \times E^{* *}$. These two results enable us to give two partial converses to Theorem 6.1 in Corollaries 6.8 and 6.13, namely that if $S$ is maximally monotone and surjective then $S$ is quasidense and that if $E$ is reflexive and $S$ is maximally monotone then $S$ is quasidense. Theorem 6.12 is particularly significant because it shows that a maximally monotone multifunction $S$ is quasidense exactly when it is of type (NI).

In Section 7, we prove the Sum theorem with domain constraints that was established in [18]. It is important to realize that we do not merely give sufficient conditions for a sum theorem for a pair of maximally monotone multifunctions to hold. In fact, we prove that, under the given conditions, the sum of a pair of closed, monotone and quasidense multifunctions is again closed, monotone and quasidense.

In Section 8, we discuss the Fitzpatrick extension of a closed, monotone and quasidense multifunction. This will be needed for our analysis of the Sum theorem with range constraints that will be the topic of Section 9. If $S: E \rightrightarrows E^{*}$ is closed, monotone and quasidense then the Fitzpatrick extension, $S^{\mathbb{F}}: E^{*} \rightrightarrows E^{* *}$, of $S$ is defined formally in terms of $\varphi_{S}{ }^{*}$ in (8.1), and we give two other characterization of $S^{\mathbb{F}}$ in (8.2). We prove in Theorem 8.2 that $S^{\mathbb{F}}$ is maximally monotone, but we will see in Example 8.8, Theorems 11.4(b) and 12.3 that it may fail to be quasidense. (It is observed in Remark 8.4, that $\left(y^{*}, y^{* *}\right) \in G\left(S^{\mathbb{F}}\right)$ exactly when $\left(y^{* *}, y^{*}\right)$ is in the Gossez extension of $\left.G(S)\right)$. $S^{\mathbb{F}}$ is defined in rather an abstract fashion, but we give a situation in Theorem 8.5 in which we can give a more explicit description of $S^{\mathbb{F}}$. Theorem 8.5 was obtained by analyzing some results of Bueno and Svaiter on linear multifunctions, which we will discuss in greater detail in Section 11. Theorem 8.5 does not have any linearity assumptions, but Theorem 8.7 is an application to linear maps.

In Section 9, we prove the Sum theorem with range constraints that was first established in [18].

In Section 10, we discuss a slight modification of an example due to Bueno and Svaiter of a nonquasidense maximally monotone skew linear operator from a subspace of $c_{0}$ into $\ell_{1}$. In Section 11 we discuss a procedure due to Bueno and Svaiter for constructing quasidense linear maps from a Banach 
space into its dual with a non-quasidense Fitzpatrick extension. In Section 12, we give a specific example of the construction of Section 11, a map from $c_{0}$ into $\ell_{1}$.

Given a maximally monotone multifunction, there are a number of conditions that are equivalent to its quasidensity. Broadly speaking, they separate into two classes, depending on whether or not they use the bidual in their definition.

Conditions that do not use the bidual include the negative alignment condition (see [18, Theorem 11.6, p. 1045]), two "fuzzy" criteria for quasidensity (in which an element of $E^{*}$ is replaced by a nonempty $w\left(E^{*}, E\right)$-compact convex subset of $E^{*}$, or an element of $E$ is replaced by a nonempty $w\left(E, E^{*}\right)$-compact convex subset of $E-$ see [19, Section 8, pp. 14-17]) and the type (FP) condition (see [19, Section 10, pp. 20-22]).

There are many classes of maximally monotone multifunctions coinciding with those of type (FP) in the literature that do require the bidual in their definitions. We mention type $(D)$, dense type, type $(E D)$ and Type (NI). These equivalences have been known for some time. See [19, Introduction, pp. 6-7] for a discusion of these with references to the sources of these results.

The bidual is not mentioned explicitly in the statements of Theorem 5.2, Corollary 6.8 or Theorem 7.3, but our proofs of all of these results ultimately depend on the bidual at one point or another. This raises the fascinating question whether there are proofs of any of these results that do not depend on the bidual. This seems to be quite a challenge. Another similar challenge is to find a proof that does not depend on the bidual of the fact that a maximally monotone multifunction is quasidense if, and only if, it is of type (FP). Of course, such a proof could not go through the equivalence of both of these classes of multifunctions with those of type (NI).

It was proved in [18, Theorem 11.9, pp. 1045-1046] that every closed, monotone quasidense multifunction is of type (ANA). It was also proved in [19, Theorem 7.2, p. 14 and Theorem 8.5, pp. 16-17] that every closed, monotone quasidense multifunction is of type (FPV), and strongly maximal. These observations lead to the three interesting problems of finding maximally monotone multifunctions that fail to be in any of these three classes.

The author would like to thank Orestes Bueno for a very interesting discussion, which led to the analysis that we present in Theorem 8.5 and Sections 11 and 12. This discussion took place during the author's stay in the Erwin Schrodinger International Institute for Mathematics and Physics of the University of Vienna in January-February, 2019. The author would like to express his sincere appreciation to the Erwin Schrodinger Institute for their support. The author would also like to thank Heinz Bauschke for reading a preliminary version of this paper and making a number of very constructive suggestion

All vector spaces in this paper are real.

\section{Fenchel Conjugates}

We start off by introducing some Banach space notation. If $X$ is a nonzero Banach space and $f: X \rightarrow$ ]$-\infty, \infty]$, we write $\operatorname{dom} f$ for $\{x \in X: f(x) \in \mathbb{R}\}$. $\operatorname{dom} f$ is the effective domain of $f$. We say that $f$ is proper if $\operatorname{dom} f \neq \emptyset$. We write $\mathscr{P} \mathscr{C}(X)$ for the set of all proper convex functions from $X$ into $]-\infty, \infty]$ and $\mathscr{P} \mathscr{C} \mathscr{L} \mathscr{S} \mathscr{C}(X)$ for the set of all proper convex lower semicontinuous functions from $X$ into $]-\infty, \infty]$. We write $X^{*}$ for the dual space of $X$ (with the pairing $\langle\cdot, \cdot\rangle: X \times X^{*} \rightarrow \mathbb{R}$ ). If $f \in \mathscr{P} \mathscr{C}(X)$, then, as usual, we define the Fenchel conjugate, $f^{*}$, of $f$ to be the function on $X^{*}$ given by

$$
f^{*}\left(x^{*}\right):=\sup _{X}\left[x^{*}-f\right]=\sup _{x \in X}\left[\left\langle x, x^{*}\right\rangle-f(x)\right] .
$$

$X^{* *}$ stands for the bidual of $X$ (with the pairing $\langle\cdot, \cdot\rangle: X^{*} \times X^{* *} \rightarrow \mathbb{R}$ ). If $g \in \mathscr{P} \mathscr{C} \mathscr{L} \mathscr{S} \mathscr{C}\left(X^{*}\right)$ then, according to (2.1), we define the Fenchel conjugate, $g^{*}$, of $g$ to be the function on $X^{* *}$ given by

$$
g^{*}\left(x^{* *}\right):=\sup _{X^{*}}\left[x^{* *}-g\right]=\sup _{x^{*} \in X^{*}}\left[\left\langle x^{*}, x^{* *}\right\rangle-g\left(x^{*}\right)\right]
$$


So, if $f \in \mathscr{P} \mathscr{C} \mathscr{L} \mathscr{S} \mathscr{C}(X)$ and we interpret $f^{* *}$ to mean $\left(f^{*}\right)^{*}$, then $f^{* *}$ is the function on $X^{* *}$ given by

$$
f^{* *}\left(x^{* *}\right):=\sup _{x^{*} \in X^{*}}\left[\left\langle x^{*}, x^{* *}\right\rangle-f^{*}\left(x^{*}\right)\right] .
$$

If $x \in X$, we write $\widehat{x}$ for the canonical image of $x$ in $X^{* *}$, that is to say

$$
\left(x, x^{*}\right) \in X \times X^{*} \Longrightarrow\left\langle x^{*}, \widehat{x}\right\rangle=\left\langle x, x^{*}\right\rangle
$$

If $g: X \rightarrow]-\infty, \infty]$, we write epi $g$ for the epigraph of $g$,

$$
\{(x, \lambda) \in X \times \mathbb{R}: g(x) \leq \lambda\} .
$$

If $h \in \mathscr{P} \mathscr{C}(X)$, the lower semicontinuous envelope of $h, \bar{h}$, is defined by epi $\bar{h}=\overline{\text { epi } h}$. See $[23$, p. 62]. Of course, to make this definition legitimate, some effort has to be made to show that $\overline{\text { epi } h}$ is the epigraph of a function. Since epi $\bar{h}$ is closed, $\bar{h}$ is lower semicontinuous. It is worth pointing out that if $h$ is a discontinuous linear functional then $\bar{h}=-\infty$ on $X$.

Theorem 2.1. Let $h \in \mathscr{P} \mathscr{C}(X)$. Let $k: X \rightarrow]-\infty, \infty]$ be lower semicontinuous and $k \leq h$ on $X$. Then $k \leq \bar{h} \leq h$ on $X$ and $\bar{h}^{*} \leq h^{*}$ on $X^{*}$. It follows from this that $\bar{h} \in \mathscr{P} \mathscr{C} \mathscr{L} \mathscr{S} \mathscr{C}(X)$ and $\bar{h}^{*}=h^{*}$ on $X^{*}$.

Proof. We know from [23, Theorem 2.2.6(i), p. 62] that $\bar{h}$ is convex. It follows from the hypotheses that epi $h \subset$ epi $k$ and epi $k$ is closed in $X \times \mathbb{R}$. Consequently, epi $h \subset$ epi $\bar{h}=\overline{\text { epi } h} \subset$ epi $k$, from which $k \leq \bar{h} \leq h$ on $X$, as required.

If $x^{*} \in X^{*}$ and $h^{*}\left(x^{*}\right) \in \mathbb{R}$ then the Fenchel-Young inequality implies that $x^{*}-h^{*}\left(x^{*}\right) \leq h$ on $X$, so epi $h \subset$ epi $\left(x^{*}-h^{*}\left(x^{*}\right)\right)$. Since $x^{*}-h^{*}\left(x^{*}\right)$ is continuous, epi $\left(x^{*}-h^{*}\left(x^{*}\right)\right)$ is closed, thus epi $\bar{h}=\overline{\mathrm{epi}} \bar{h} \subset$ epi $\left(x^{*}-h^{*}\left(x^{*}\right)\right)$, from which $\bar{h} \geq x^{*}-h^{*}\left(x^{*}\right)$ on $X$. It follows easily that $\bar{h}^{*}\left(x^{*}\right) \leq h^{*}\left(x^{*}\right)$. Of course, this inequality persists even if $h^{*}\left(x^{*}\right)=\infty$, and so we have proved that $\bar{h}^{*} \leq h^{*}$ on $X^{*}$. This completes the proof of Theorem 2.1.

The main tool in the proof of Theorem 2.1 was epigraphical analysis. The drawback of this method is that the definition of $\bar{h}$ is not very intuitive. We now discuss a more explicit geometric method of obtaining the function required for Theorem 4.11 , which we can actually express as a biconjugate. The preliminary work is done in Lemma 2.2 below, which is of independent interest.

We shall use Rockafellar's version of the Fenchel duality theorem (which originally appeared in Rockafellar, [13, Theorem 3(a), p. 85]) in the following form: Let $p, u \in \mathscr{P} \mathscr{C}(X)$ and $u$ be continuous. Then

$$
(p+u)^{*}(0)=\min _{x^{*} \in X^{*}}\left[p^{*}\left(x^{*}\right)+u^{*}\left(-x^{*}\right)\right] .
$$

We could have used instead König's sandwich theorem, a simple application of the Hahn-Banach theorem, see [8, Theorem 1.7, p. 112].

Lemma 2.2. Let $p \in \mathscr{P} \mathscr{C}(X)$. Let $s: X \rightarrow]-\infty, \infty]$ be lower semicontinuous, $s \leq p$ on $X$ and $s(0)>0$. Then:

(a) There exists $K \in[0, \infty[$ such that $p+K\|\cdot\| \geq 0$ on $X$.

(b) There exists $x^{*} \in X^{*}$ such that $p^{*}\left(x^{*}\right) \leq 0$.

Proof. (a) Since the result is obvious if $p \geq 0$ on $X$, we can and will suppose that there exists $w \in E$ such that $p(w)<0$. Let $\theta \in \mathbb{R}, \theta<s(w)$. It follows that $\theta<s(w) \leq p(w)<0$. Since $s$ is lower semicontinuous, there exists $m \geq 1$ such that $\inf _{y \in X, s(y) \leq 0}\|y\| \geq \frac{1}{m}$ and $\inf _{z \in X, s(z) \leq \theta}\|z-w\| \geq \frac{1}{m}$. Let $\alpha:=p(w)-\theta>0$. Let $y \in X$. We will show that

$$
p(y)+\alpha m^{2}\|w\|\|y\|-\theta m\|y\| \geq 0 .
$$

This gives the desired result, with $K:=\alpha m^{2}\|w\|-\theta m$.

Case 1. $(p(y) \geq 0)$ In this case, (2.4) is obvious since $\alpha>0$ and $\theta<0$. 
Case 2. $(\theta \leq p(y)<0)$ In this case, $s(y)<0$, and so $\|y\| \geq \frac{1}{m}$. Hence $m\|y\|-1 \geq 0$. Again since $\alpha>0$ and $\theta<0$,

$$
p(y)+\alpha m^{2}\|w\|\|y\|-\theta m\|y\| \geq \theta-\theta m\|y\|=(-\theta)(m\|y\|-1) \geq 0,
$$

which gives (2.4).

Case 3. $(p(y)<\theta)$ Let $\beta:=\theta-p(y)>0$. $\beta$ (unlike $\alpha$ ) depends on $y$. Here, the convexity of $p$ and the fact that $s \leq p$ on $X$ imply that

$$
s\left(\frac{\alpha y+\beta w}{\alpha+\beta}\right) \leq p\left(\frac{\alpha y+\beta w}{\alpha+\beta}\right) \leq \frac{\alpha p(y)+\beta p(w)}{\alpha+\beta}=\frac{\alpha(\theta-\beta)+\beta(\alpha+\theta)}{\alpha+\beta}=\theta .
$$

Thus, from the choice of $m$ again,

$$
\frac{\alpha(\|y\|+\|w\|)}{\alpha+\beta} \geq\left\|\frac{\alpha(y-w)}{\alpha+\beta}\right\|=\left\|\frac{\alpha y+\beta w}{\alpha+\beta}-w\right\| \geq \frac{1}{m} .
$$

This is equivalent to the statement $\alpha m\|w\|+\alpha m\|y\|-\alpha-\beta \geq 0$. Substituting $\beta=\theta-p(y)$, we see that

$$
p(y) \geq \theta+\alpha-\alpha m\|w\|-\alpha m\|y\| \text {. }
$$

We still have $m\|y\|-1 \geq 0$, and also $\alpha m\|w\|-\theta-\alpha=\alpha m\|w\|-p(w)>0$. It follows that $(\alpha m\|w\|-\theta-\alpha)(m\|y\|-1) \geq 0$. Equivalently,

$$
\alpha m^{2}\|w\|\|y\|-\theta m\|y\| \geq \alpha m\|y\|+\alpha m\|w\|-\theta-\alpha .
$$

(2.4) now follows by adding this to (2.5).

Now let $u:=K\|\cdot\|$. From (a), $p+u \geq 0$ on $X$, and so $(p+u)^{*}(0) \leq 0$. (2.3) now gives $x^{*} \in X^{*}$ such that $p^{*}\left(x^{*}\right)+u^{*}\left(-x^{*}\right) \leq 0$. Since $u(0)=0, u^{*}\left(-x^{*}\right) \geq 0$, and thus we obtain (b).

Theorem 2.3. Let $h \in \mathscr{P} \mathscr{C}(X)$. Let $k: X \rightarrow]-\infty, \infty]$, be lower semicontinuous and $k \leq h$ on $X$. For all $x \in X$, let $f(x):=\sup _{x^{*} \in X^{*}}\left[\left\langle x, x^{*}\right\rangle-h^{*}\left(x^{*}\right)\right]$, i.e., $f(x):=h^{* *}(\widehat{x})$. Then:

(a) $f \geq k$ on $X$, and so $f: X \rightarrow]-\infty, \infty]$.

(b) $f \in \mathscr{P} \mathscr{C} \mathscr{L} \mathscr{S} \mathscr{C}(X)$ and $f^{*}=h^{*}$ on $X^{*}$.

Proof. (a) Let $x \in X, \lambda \in \mathbb{R}$ and $\lambda<k(x)$. Let $p(y):=h(y+x)-\lambda$ and $s(y):=k(y+x)-\lambda$, so $s(0)=k(x)-\lambda>0$. Lemma 2.2(b) now gives $x^{*} \in X^{*}$ such that $p^{*}\left(x^{*}\right) \leq 0$. It is easily seen that this is equivalent to the statement that $\left\langle x, x^{*}\right\rangle-h^{*}\left(x^{*}\right) \geq \lambda$. (a) now follows by letting $\lambda \rightarrow k(x)$.

(b) From the Fenchel-Young inequality, for all $x^{*} \in X^{*}, x^{*}-h^{*}\left(x^{*}\right) \leq h$ on $X$, thus $f \leq h$ on $X$, and so $f \in \mathscr{P} \mathscr{C} \mathscr{L} \mathscr{S} \mathscr{C}(X)$ and $f^{*} \geq h^{*}$ on $X^{*}$. On the other hand, for all $x^{*} \in X^{*}, f \geq x^{*}-h^{*}\left(x^{*}\right)$ on $X$, i.e., $x^{*}-f \leq h^{*}\left(x^{*}\right)$ on $X$ thus, for all $x^{*} \in X^{*}, f^{*}\left(x^{*}\right)=\sup _{X}\left[x^{*}-f\right] \leq h^{*}\left(x^{*}\right)$ on $X$. Thus $f^{*}=h^{*}$ on $X^{*}$, completing the proof of (b).

\section{3. $E \times E^{*}, q_{L}, r_{L}$ AND QUASIDENSITY}

Now let $E$ be nonzero Banach space. For all $\left(x, x^{*}\right) \in E \times E^{*}$, let $\quad\left\|\left(x, x^{*}\right)\right\|:=\sqrt{\|x\|^{2}+\left\|x^{*}\right\|^{2}}$, and represent $\left(E \times E^{*}\right)^{*}$ by $E^{*} \times E^{* *}$, under the pairing

$$
\left\langle\left(x, x^{*}\right),\left(y^{*}, y^{* *}\right)\right\rangle:=\left\langle x, y^{*}\right\rangle+\left\langle x^{*}, y^{* *}\right\rangle .
$$

Define the linear map $L: E \times E^{*} \rightarrow E^{*} \times E^{* *}$ by

$$
L\left(x, x^{*}\right):=\left(x^{*}, \widehat{x}\right) .
$$

Then

$$
\text { for all } a, b \in E \times E^{*}, \quad\langle a, L b\rangle=\langle b, L a\rangle .
$$

We define the even real functions $q_{L}$ and $r_{L}$ on $E \times E^{*}$ by $q_{L}\left(x, x^{*}\right):=\left\langle x, x^{*}\right\rangle$ and

$$
r_{L}\left(x, x^{*}\right):=\frac{1}{2}\|x\|^{2}+\frac{1}{2}\left\|x^{*}\right\|^{2}+\left\langle x, x^{*}\right\rangle=\frac{1}{2}\left\|\left(x, x^{*}\right)\right\|^{2}+q_{L}\left(x, x^{*}\right) .
$$


For all $\left(x, x^{*}\right) \in E \times E^{*}, \quad\left|q_{L}\left(x, x^{*}\right)\right|=\left|\left\langle x, x^{*}\right\rangle\right| \leq\|x\|\left\|x^{*}\right\| \leq \frac{1}{2}\left\|\left(x, x^{*}\right)\right\|^{2}, \quad$ so

$$
0 \leq r_{L} \leq\|\cdot\|^{2} \text { on } E \times E^{*} \text {. }
$$

We note for future reference that,

$$
\text { for all } b, c \in E \times E^{*}, \quad q_{L}(b-c)=q_{L}(b)+q_{L}(c)-\langle b, L c\rangle .
$$

Definition 3.1. Let $A \subset E \times E^{*}$. We say that $A$ is quasidense (in $E \times E^{*}$ ) if

$$
c \in E \times E^{*} \Longrightarrow \inf r_{L}(A-c) \leq 0 \Longleftrightarrow \inf r_{L}(A-c)=0 \text {. }
$$

(The " $\Longleftrightarrow "$ above follows since $r_{L} \geq 0$.) In longhand, (3.5) can be rewritten:

$$
\left(x, x^{*}\right) \in E \times E^{*} \Longrightarrow \inf _{\left(s, s^{*}\right) \in A}\left[\frac{1}{2}\|s-x\|^{2}+\frac{1}{2}\left\|s^{*}-x^{*}\right\|^{2}+\left\langle s-x, s^{*}-x^{*}\right\rangle\right] \leq 0 .
$$

Example 3.2 (Subdifferentials). Let $f: E \rightarrow]-\infty, \infty]$ be proper, convex and lower semicontinuous and $\partial f$ be the usual subdifferential. Then $G(\partial f)$ is quasidense. There is an "elementary" proof of this in [19, Theorem 4.6]. There is also a more sophisticated proof based on Theorem 4.8 below in [18, Theorem 7.5, p. 1033]. We shall see in Theorem 6.1 below that this result generalizes Rockafellar's maximal monotonicity theorem.

In fact, the "elementary" proof mentioned above can be generalized to some more general subdifferentials for non-convex functions. See Simons-Wang, [20, Definition 2.1, p. 633] and [20, Theorem 3.2, pp. 634-635].

The dual norm on $E^{*} \times E^{* *}$ is given by $\left\|\left(y^{*}, y^{* *}\right)\right\|:=\sqrt{\left\|y^{*}\right\|^{2}+\left\|y^{* *}\right\|^{2}}$. Define the linear map $\widetilde{L}: \quad E^{*} \times E^{* *} \rightarrow E^{* *} \times E^{* * *}$ by $\widetilde{L}\left(x^{*}, x^{* *}\right):=\left(x^{* *}, \widehat{x^{*}}\right)$. Then $q_{\widetilde{L}}\left(y^{*}, y^{* *}\right):=\left\langle y^{*}, y^{* *}\right\rangle$ and $r_{\widetilde{L}}\left(y^{*}, y^{* *}\right):=$ $\frac{1}{2}\left\|y^{*}\right\|^{2}+\frac{1}{2}\left\|y^{* *}\right\|^{2}+\left\langle y^{*}, y^{* *}\right\rangle=\frac{1}{2}\left\|\left(y^{*}, y^{* *}\right)\right\|^{2}+q_{\widetilde{L}}\left(y^{*}, y^{* *}\right)$.

One can easily verify the following generalization of (3.4):

$$
c \in E \times E^{*} \text { and } c^{*} \in E^{*} \times E^{* *} \Longrightarrow q_{\widetilde{L}}\left(c^{*}+L c\right)=q_{\widetilde{L}}\left(c^{*}\right)+\left\langle c, c^{*}\right\rangle+q_{L}(c) .
$$

Lemma 3.3 below gives a very nice relationship between $L$ and quasidensity. It is the first of two preliminary results leading to the main result of this section, Theorem 3.5.

Lemma 3.3. $L\left(E \times E^{*}\right)$ is quasidense in $E^{*} \times E^{* *}$. In other words:

$$
c^{*} \in E^{*} \times E^{* *} \Longrightarrow \inf _{c \in E \times E^{*}} r_{\widetilde{L}}\left(L c-c^{*}\right)=0 .
$$

In longhand, this can be rewritten: for all $\left(y^{*}, y^{* *}\right) \in E^{*} \times E^{* *}$,

$$
\inf _{\left(x, x^{*}\right) \in E \times E^{*}}\left[\frac{1}{2}\left\|y^{*}-x^{*}\right\|^{2}+\frac{1}{2}\left\|y^{* *}-\widehat{x}\right\|^{2}+\left\langle y^{*}-x^{*}, y^{* *}-\widehat{x}\right\rangle\right]=0 .
$$

Proof. Let $\left(y^{*}, y^{* *}\right) \in E^{*} \times E^{* *}$. For all $\varepsilon>0$, the definition of $\left\|y^{* *}\right\|$ provides $z^{*} \in E^{*}$ such that $\left\|z^{*}\right\| \leq$ $\left\|y^{* *}\right\|$ and $\left\langle z^{*}, y^{* *}\right\rangle \leq-\left\|y^{* *}\right\|^{2}+\varepsilon$, from which $\frac{1}{2}\left\|z^{*}\right\|^{2}+\frac{1}{2}\left\|y^{* *}\right\|^{2}+\left\langle z^{*}, y^{* *}\right\rangle \leq\left\|y^{* *}\right\|^{2}+\left\langle z^{*}, y^{* *}\right\rangle \leq \varepsilon$. So

$$
\begin{aligned}
0 & \leq \inf _{\left(x, x^{*}\right) \in E \times E^{*}}\left[\frac{1}{2}\left\|y^{*}-x^{*}\right\|^{2}+\frac{1}{2}\left\|y^{* *}-\widehat{x}\right\|^{2}+\left\langle y^{*}-x^{*}, y^{* *}-\widehat{x}\right\rangle\right] \\
& =\inf _{\left(x, z^{*}\right) \in E \times E^{*}}\left[\frac{1}{2}\left\|z^{*}\right\|^{2}+\frac{1}{2}\left\|y^{* *}-\widehat{x}\right\|^{2}+\left\langle z^{*}, y^{* *}-\widehat{x}\right\rangle\right] \\
& \leq \inf _{z^{*} \in E^{*}}\left[\frac{1}{2}\left\|z^{*}\right\|^{2}+\frac{1}{2}\left\|y^{* *}\right\|^{2}+\left\langle z^{*}, y^{* *}\right\rangle\right] \leq 0 .
\end{aligned}
$$

This establishes (3.9), and hence (3.8).

Lemma 3.4. Let $b \in E \times E^{*}$ and $b^{*} \in E^{*} \times E^{* *}$. Then

$$
q_{\widetilde{L}}\left(L b+b^{*}\right) \leq r_{L}(b)+r_{\widetilde{L}}\left(b^{*}\right) .
$$

Let $a, c \in E \times E^{*}$ and $c^{*} \in E^{*} \times E^{* *}$. Then

$$
q_{\widetilde{L}}\left(L a-c^{*}\right) \leq r_{L}(a-c)+r_{\widetilde{L}}\left(L c-c^{*}\right) .
$$


Proof. From (3.7),

$$
\begin{gathered}
r_{L}(b)+r_{\widetilde{L}}\left(b^{*}\right)-q_{\widetilde{L}}\left(L b+b^{*}\right) \\
=q_{L}(b)+\frac{1}{2}\|b\|^{2}+q_{\widetilde{L}}\left(b^{*}\right)+\frac{1}{2}\left\|b^{*}\right\|^{2}-q_{L}(b)-\left\langle b, b^{*}\right\rangle-q_{\widetilde{L}}\left(b^{*}\right) \\
=\frac{1}{2}\|b\|^{2}+\frac{1}{2}\left\|b^{*}\right\|^{2}-\left\langle b, b^{*}\right\rangle \geq \frac{1}{2}\|b\|^{2}+\frac{1}{2}\left\|b^{*}\right\|^{2}-\|b\|\left\|b^{*}\right\| \geq 0 .
\end{gathered}
$$

This completes the proof of (3.10), and (3.11) follows from (3.10) with $b:=a-c$ and $b^{*}:=L c-c^{*}$

We have the following fundamental result:

Theorem 3.5. Let $A \subset E \times E^{*}$ and $A$ be quasidense in $E \times E^{*}$. Then, for all $c^{*} \in E^{*} \times E^{* *}$, $\inf q_{\widetilde{L}}\left(L(A)-c^{*}\right) \leq 0$.

Proof. Let $c^{*} \in E^{*} \times E^{* *}$ and $\varepsilon>0$. Then, from Lemma 3.3 and Definition 3.1, there exist $c \in E \times E^{*}$ and then $a \in A$ such that $r_{\widetilde{L}}\left(L c-c^{*}\right)<\frac{1}{2} \varepsilon$ and $r_{L}(a-c)<\frac{1}{2} \varepsilon$. From (3.11), $q_{\widetilde{L}}\left(L a-c^{*}\right)<\varepsilon$.

The following definition was made in [14, Definition 10, p. 183]:

Definition 3.6. Let $A \subset E \times E^{*}$. Then $A$ is of type (NI) if,

$$
\text { for all }\left(y^{*}, y^{* *}\right) \in E^{*} \times E^{* *}, \quad \inf _{\left(s, s^{*}\right) \in A}\left\langle s^{*}-y^{*}, \widehat{s}-y^{* *}\right\rangle \leq 0 .
$$

In our current notation, (3.12) can be rephrased as

$$
\text { for all } c^{*} \in E^{*} \times E^{* *}, \quad \inf _{a \in A} q_{\widetilde{L}}\left(L a-c^{*}\right) \leq 0 .
$$

"(NI)" stands for "negative infimum". We note that $A$ is not constrained to be monotone in this definition.

Corollary 3.7. Let $A \subset E \times E^{*}$ and $A$ be quasidense in $E \times E^{*}$. Then $A$ is of type (NI).

Proof. This is immediate from Theorem 3.5 and (3.13).

There is another way of viewing Theorem 3.5. In order to explain this, we introduce the function $\Theta_{A}$. (Compare [18, Definition 6.2, p. 1029].)

Definition 3.8. Let $A \subset E \times E^{*}$ and $A \neq \emptyset$. We define the function $\left.\left.\Theta_{A}: E^{*} \times E^{* *} \rightarrow\right]-\infty, \infty\right]$ by:

$$
\text { for all } c^{*} \in E^{*} \times E^{* *}, \quad \Theta_{A}\left(c^{*}\right):=\sup _{A}\left[c^{*}-q_{L}\right]=\sup _{a \in A}\left[\left\langle a, c^{*}\right\rangle-q_{L}(a)\right] \text {. }
$$

In longhand: for all $\left(y^{*}, y^{* *}\right) \in E^{*} \times E^{* *}$,

$$
\Theta_{A}\left(y^{*}, y^{* *}\right):=\sup _{\left(s, s^{*}\right) \in A}\left[\left\langle s, y^{*}\right\rangle+\left\langle s^{*}, y^{* *}\right\rangle-\left\langle s, s^{*}\right\rangle\right] .
$$

Corollary 3.9. Let $A \subset E \times E^{*}$ and $A$ be quasidense in $E \times E^{*}$. Then $\Theta_{A} \geq q_{\widetilde{L}}$ on $E^{*} \times E^{* *}$.

Proof. Let $c^{*} \in E^{*} \times E^{* *}$. Then, from Definition 3.8 and (3.7),

$$
\begin{aligned}
\Theta_{A}\left(c^{*}\right)-q_{\widetilde{L}}\left(c^{*}\right) & =\sup _{a \in A}\left[\left\langle a, c^{*}\right\rangle-q_{L}(a)-q_{\widetilde{L}}\left(c^{*}\right)\right] \\
& =-\inf _{a \in A}\left[q_{\widetilde{L}}\left(c^{*}\right)-\left\langle a, c^{*}\right\rangle+q_{L}(a)\right] \\
& =-\inf _{a \in A} q_{\widetilde{L}}\left(L a-c^{*}\right) .
\end{aligned}
$$

The result now follows since, from Theorem 3.5, $\inf _{a \in A} q_{\widetilde{L}}\left(L a-c^{*}\right) \leq 0$.

Remark 3.10. Corollary 3.9 will be used in Lemma 4.7, Theorem 6.12 and Theorem 11.3. The converses of Corollaries 3.7 and 3.9 are true for maximally monotone sets. (See Theorem 6.12). We give an example where the converse of Corollary 3.7 fails without the hypothesis of maximal monotonicity in Example 3.12 below. Example 3.12 depends on the following simple fact:

Fact 3.11. Let $E$ be reflexive, $S: E \rightrightarrows E^{*}, D(S)=E$ and $A:=G(S)$. Then $A$ is of type (NI). 
Proof. Let $\left(y^{*}, y^{* *}\right) \in E^{*} \times E^{* *}$. Since $E$ is reflexive, there exists $s \in E$ such that $\widehat{s}=y^{* *}$. Since $D(S)=E$ and $G(S)=A$, there exists $s^{*} \in E^{*}$ such that $\left(s, s^{*}\right) \in A$. Since $\left\langle s^{*}-y^{*}, \widehat{s}-y^{* *}\right\rangle=\left\langle s^{*}-y^{*}, 0\right\rangle=0, A$ is of type (NI).

Example 3.12. Let $E=\mathbb{R}$. If $\left(s, s^{*}\right),\left(x, x^{*}\right) \in \mathbb{R} \times \mathbb{R}$ then

$$
\begin{aligned}
r_{L}\left(\left(s, s^{*}\right)-\left(x, x^{*}\right)\right) & =\frac{1}{2}\|s-x\|^{2}+\frac{1}{2}\left\|s^{*}-x^{*}\right\|^{2}+\left\langle s-x, s^{*}-x^{*}\right\rangle \\
& =\frac{1}{2}(s-x)^{2}+\frac{1}{2}\left(s^{*}-x^{*}\right)^{2}+(s-x)\left(s^{*}-x^{*}\right) \\
& =\frac{1}{2}\left(s+s^{*}-x-x^{*}\right)^{2}
\end{aligned}
$$

Let $A:=\{(\lambda,-\lambda): \lambda \in \mathbb{R}\} \subset \mathbb{R} \times \mathbb{R}$ and $\left(x, x^{*}\right):=(1,0) \in \mathbb{R} \times \mathbb{R}$ then, for all $\left(s, s^{*}\right) \in A$, $r_{L}\left(\left(s, s^{*}\right)-(1,0)\right)=\frac{1}{2}(s-s-1-0)^{2}=\frac{1}{2}$. Thus $A$ is not quasidense. However, from Fact 3.11, $A$ is of type $(\mathrm{NI})$.

\section{QUASIDENSE SETS DETERMINED BY THE COINCIDENCE SETS OF CONVEX FUNCTIONS}

Definition 4.1. If $f \in \mathscr{P} \mathscr{C}\left(E \times E^{*}\right)$ and $f \geq q_{L}$ on $E \times E^{*}$, we write coinc $[f]$ for the "coincidence set"

$$
\left\{b \in E \times E^{*}: f(b)=q_{L}(b)\right\} .
$$

The notation " $M_{f}$ " has been used for this set in the literature. We have avoided the " $M_{f}$ " notation because it lead to superscripts and subscripts on subscripts, and consequently makes the analysis harder to read. If $g$ is a proper, convex function on $E^{*} \times E^{* *}$ and $g \geq q_{\widetilde{L}}$ on $E^{*} \times E^{* *}$, we write dcoinc $[g]$ for the "dual coincidence set"

$$
\left\{b^{*} \in E^{*} \times E^{* *}: g\left(b^{*}\right)=q_{\widetilde{L}}\left(b^{*}\right)\right\} .
$$

Lemmas 4.2 and 4.3 lead to the main result of the section, Theorem 4.4:

Lemma 4.2 (A boundedness result). Let $X$ be a nonzero real Banach space and $g \in \mathscr{P} \mathscr{C}(X)$. Suppose, further, that $\inf _{x \in X}\left[g(x)+\frac{1}{2}\|x\|^{2}\right]=0, y, z \in X, g(y)+\frac{1}{2}\|y\|^{2} \leq 1$ and $g(z)+\frac{1}{2}\|z\|^{2} \leq 1$. Then $\|y\| \leq\|z\|+\sqrt{8}$.

Proof. We have $\frac{1}{8}[\|y\|-\|z\|]^{2}=\frac{1}{4}\|y\|^{2}+\frac{1}{4}\|z\|^{2}-\frac{1}{8}[\|y\|+\|z\|]^{2}$ and

$$
0 \leq g\left(\frac{1}{2} y+\frac{1}{2} z\right)+\frac{1}{2}\left\|\frac{1}{2} y+\frac{1}{2} z\right\|^{2} \leq \frac{1}{2} g(y)+\frac{1}{2} g(z)+\frac{1}{8}[\|y\|+\|z\|]^{2} .
$$

Thus, by addition,

$$
\frac{1}{8}[\|y\|-\|z\|]^{2} \leq \frac{1}{2} g(y)+\frac{1}{2} g(z)+\frac{1}{4}\|y\|^{2}+\frac{1}{4}\|z\|^{2} \leq \frac{1}{2}+\frac{1}{2}=1 .
$$

This gives the required result.

Lemma 4.3. Let $b, d \in E \times E^{*}$. Then:

$$
r_{L}(b+d) \leq r_{L}(b)+2\|b\|\|d\|+r_{L}(d) \leq\|b\|^{2}+2\|b\|\|d\|+r_{L}(d) .
$$

Proof. Let $b=\left(x, x^{*}\right)$ and $d=\left(z, z^{*}\right)$. From the Cauchy-Schwarz inequality, we have

$$
\|x\|\|z\|+\left\|x^{*}\right\|\left\|z^{*}\right\| \leq \sqrt{\|x\|^{2}+\left\|x^{*}\right\|^{2}} \sqrt{\|z\|^{2}+\left\|z^{*}\right\|^{2}}=\|b\|\|d\| .
$$

From the triangle inequality, we have

$$
\|x+z\|^{2} \leq(\|x\|+\|z\|)^{2}=\|x\|^{2}+2\|x\|\|z\|+\|z\|^{2}
$$

and

$$
\left\|x^{*}+z^{*}\right\|^{2} \leq\left(\left\|x^{*}\right\|+\left\|z^{*}\right\|\right)^{2}=\left\|x^{*}\right\|^{2}+2\left\|x^{*}\right\|\left\|z^{*}\right\|+\left\|z^{*}\right\|^{2} .
$$


Thus

$$
\begin{aligned}
\frac{1}{2}\|b+d\|^{2} & =\frac{1}{2}\|x+z\|^{2}+\frac{1}{2}\left\|x^{*}+z^{*}\right\|^{2} \\
& \leq \frac{1}{2}\|x\|^{2}+\|x\|\|z\|+\frac{1}{2}\|z\|^{2}+\frac{1}{2}\left\|x^{*}\right\|^{2}+\left\|x^{*}\right\|\left\|z^{*}\right\|+\frac{1}{2}\left\|z^{*}\right\|^{2} \\
& \leq \frac{1}{2}\|b\|^{2}+\|b\|\|d\|+\frac{1}{2}\|d\|^{2} .
\end{aligned}
$$

Also, from (3.4) with $c:=-d$ and the fact that $\|L d\|=\|d\|$,

$$
q_{L}(b+d)=q_{L}(b)+\langle b, L d\rangle+q_{L}(d) \leq q_{L}(b)+\|b\|\|d\|+q_{L}(d) .
$$

The result now follows by addition, (3.2) and (3.3).

Theorem 4.4 (Primal condition for quasidensity). Let $f \in \mathscr{P} \mathscr{C} \mathscr{L} \mathscr{S} \mathscr{C}\left(E \times E^{*}\right)$ and $f \geq q_{L}$ on $E \times E^{*}$. For all $c, b \in E \times E^{*}$, let

$$
f_{c}(b):=f(b+c)-\langle b, L c\rangle-q_{L}(c)=\left(f-q_{L}\right)(b+c)+q_{L}(b) \geq q_{L}(b) .
$$

(The first expression shows that $f_{c} \in \mathscr{P} \mathscr{C} \mathscr{L} \mathscr{S} \mathscr{C}\left(E \times E^{*}\right)$.) Then (a) $\Longleftrightarrow$ (b):

(a) coinc $[f]$ is quasidense.

(b) For all $c \in E \times E^{*}, \inf _{b \in E \times E^{*}}\left[f_{c}(b)+\frac{1}{2}\|b\|^{2}\right] \leq 0$.

Proof. Let $A:=\operatorname{coinc}[f]$. Let $c \in E \times E^{*}$. Since $f_{c}=q_{L}$ on $A-c$,

$$
\begin{aligned}
\inf _{b \in E \times E^{*}}\left[f_{c}(b)+\frac{1}{2}\|b\|^{2}\right] & \leq \inf _{b \in A-c}\left[f_{c}(b)+\frac{1}{2}\|b\|^{2}\right] \\
& =\inf _{b \in A-c}\left[q_{L}(b)+\frac{1}{2}\|b\|^{2}\right] \\
& =\inf _{b \in A-c} r_{L}(b)=\inf r_{L}(A-c)=0,
\end{aligned}
$$

and so it follows that (a) $\Longrightarrow$ (b).

Suppose now that (b) is satisfied and $c \in E \times E^{*}$. Let $c_{0}:=c$, so that $\inf _{b \in E \times E^{*}}\left[f_{c_{0}}(b)+\frac{1}{2}\|b\|^{2}\right] \leq 0$. From (4.1), $f_{c_{0}}+\frac{1}{2}\|\cdot\|^{2} \geq q_{L}+\frac{1}{2}\|\cdot\|^{2} \geq 0$ on $E \times E^{*}$, so in fact $\inf _{b \in E \times E^{*}}\left[f_{c_{0}}(b)+\frac{1}{2}\|b\|^{2}\right]=0$. From Lemma 4.2, there exists $M \in \mathbb{R}$ such that

$$
f_{c_{0}}(b)+\frac{1}{2}\|b\|^{2} \leq 1 \quad \Longrightarrow \quad\|b\| \leq M \text {. }
$$

Let $0 \leq \varepsilon<1$. Let $1 \geq \varepsilon_{1} \geq \varepsilon_{3} \geq \varepsilon_{3} \cdots>0$ and $\sum_{n=1}^{\infty} \varepsilon_{n} \leq \varepsilon$. We now define inductively $c_{1}, c_{2}, \cdots \in$ $E \times E^{*}$. Suppose that $n \geq 0$ and $c_{n}$ is known. By hypohesis, $\inf _{b \in E \times E^{*}}\left[f_{c_{n}}(b)+\frac{1}{2}\|b\|^{2}\right] \leq 0$, and so there exists $b_{n} \in E \times E^{*}$ such that $f_{c_{n}}\left(b_{n}\right)+\frac{1}{2}\left\|b_{n}\right\|^{2} \leq \varepsilon_{n+1}^{2}$. Let $c_{n+1}:=b_{n}+c_{n}$. This completes the inductive construction.

Since $b_{n}=c_{n+1}-c_{n}$, we now have $c_{0}, c_{1}, c_{2}, \ldots$, such that,

$$
\text { for all } n \geq 0, \quad f_{c_{n}}\left(c_{n+1}-c_{n}\right)+\frac{1}{2}\left\|c_{n+1}-c_{n}\right\|^{2} \leq \varepsilon_{n+1}^{2} .
$$

From (4.1), $f_{c_{n}}\left(c_{n+1}-c_{n}\right)=\left(f-q_{L}\right)\left(c_{n+1}\right)+q_{L}\left(c_{n+1}-c_{n}\right)$ and so,

$$
\text { for all } n \geq 0, \quad\left(f-q_{L}\right)\left(c_{n+1}\right)+r_{L}\left(c_{n+1}-c_{n}\right) \leq \varepsilon_{n+1}^{2} .
$$

Since $f \geq q_{L}$ and, from (3.3), $r_{L} \geq 0$ on $E \times E^{*}$, this implies that,

$$
\text { for all } n \geq 0, \quad\left(f-q_{L}\right)\left(c_{n+1}\right) \leq \varepsilon_{n+1}^{2} \quad \text { and } \quad r_{L}\left(c_{n+1}-c_{n}\right) \leq \varepsilon_{n+1}^{2} .
$$

We now prove that,

$$
\text { for all } n \geq 1, \quad\left\|c_{n+1}-c_{n}\right\| \leq \sqrt{10} \varepsilon_{n} .
$$

Let $n \geq 1$. Since $f$ is convex, (4.4) gives

$$
2 f\left(\frac{1}{2} c_{n+1}+\frac{1}{2} c_{n}\right) \leq f\left(c_{n+1}\right)+f\left(c_{n}\right) \leq q_{L}\left(c_{n+1}\right)+\varepsilon_{n+1}^{2}+q_{L}\left(c_{n}\right)+\varepsilon_{n}^{2} .
$$

Since $f \geq q_{L}$ on $E \times E^{*}$ and $\varepsilon_{n+1}^{2} \leq \varepsilon_{n}^{2}$, it follows that

$$
2 q_{L}\left(\frac{1}{2} c_{n+1}+\frac{1}{2} c_{n}\right)-q_{L}\left(c_{n+1}\right)-q_{L}\left(c_{n}\right) \leq 2 \varepsilon_{n}^{2} .
$$


Thus, from the quadraticity of $q_{L}, \frac{1}{2} q_{L}\left(c_{n+1}+c_{n}\right)-q_{L}\left(c_{n+1}\right)-q_{L}\left(c_{n}\right) \leq 2 \varepsilon_{n}^{2}$. Since $q_{L}\left(c_{n+1}\right)+q_{L}\left(c_{n}\right)=$ $\frac{1}{2} q_{L}\left(c_{n+1}+c_{n}\right)+\frac{1}{2} q_{L}\left(c_{n+1}-c_{n}\right)$, we see that

$$
-q_{L}\left(c_{n+1}-c_{n}\right) \leq 4 \varepsilon_{n}^{2} .
$$

From (4.4), $q_{L}\left(c_{n+1}-c_{n}\right)+\frac{1}{2}\left\|c_{n+1}-c_{n}\right\|^{2}=r_{L}\left(c_{n+1}-c_{n}\right) \leq \varepsilon_{n+1}^{2}$. Thus

$$
\text { for all } n \geq 1, \quad \frac{1}{2}\left\|c_{n+1}-c_{n}\right\|^{2} \leq 4 \varepsilon_{n}^{2}+\varepsilon_{n+1}^{2} \leq 5 \varepsilon_{n}^{2} \text {. }
$$

Thus we obtain (4.5). We will also need an estimate for $\left\|c_{1}-c_{0}\right\|$. This is not covered by (4.5). Now (4.5) used the inequality $f\left(c_{n}\right) \leq q_{L}\left(c_{n}\right)+\varepsilon_{n}^{2}$. A similar analysis for $\left\|c_{1}-c_{0}\right\|$ is unlikely, because we have no knowledge about $f\left(c_{0}\right)$ - there is no a priori reason why $f\left(c_{0}\right)$ should even be finite. This issue is partially resolved by (4.7) below.

It follows from (4.5) that $\lim _{n \rightarrow \infty} c_{n}$ exists. Let $a_{\varepsilon}:=\lim _{n \rightarrow \infty} c_{n}$. Clearly, $a_{\varepsilon}-c_{1}=\sum_{n=1}^{\infty}\left(c_{n+1}-c_{n}\right)$ and so, from (4.5),

$$
\left.\begin{array}{rl}
\left\|a_{\varepsilon}-c_{1}\right\| & =\left\|\sum_{n=1}^{\infty}\left(c_{n+1}-c_{n}\right)\right\| \\
& \leq \sum_{n=1}^{\infty}\left\|c_{n+1}-c_{n}\right\| \leq \sqrt{10} \sum_{n=1}^{\infty} \varepsilon_{n} \leq 4 \varepsilon .
\end{array}\right\}
$$

From (4.4), the lower semicontinuity of $f$, and the continuity of $q_{L}, f\left(a_{\varepsilon}\right) \leq q_{L}\left(a_{\varepsilon}\right)$, and so $a_{\varepsilon} \in \operatorname{coinc}[f]$. We must now estimate $r_{L}\left(a_{\varepsilon}-c\right)$. (4.3) with $n=0$ gives $f_{c_{0}}\left(c_{1}-c_{0}\right)+\frac{1}{2}\left\|c_{1}-c_{0}\right\|^{2} \leq \varepsilon_{1}^{2} \leq 1$ and so, from (4.2),

$$
\left\|c_{1}-c\right\|=\left\|c_{1}-c_{0}\right\| \leq M
$$

Furthermore, (4.4) with $n=0$ gives

$$
r_{L}\left(c_{1}-c\right)=r_{L}\left(c_{1}-c_{0}\right) \leq \varepsilon_{1}^{2} \leq \varepsilon .
$$

From Lemma 4.3 with $b=a_{\varepsilon}-c_{1}$ and $d=c_{1}-c$, (4.6), (4.7) and (4.8),

$$
\begin{aligned}
r_{L}\left(a_{\varepsilon}-c\right) & \leq\left\|a_{\varepsilon}-c_{1}\right\|^{2}+2\left\|a_{\varepsilon}-c_{1}\right\|\left\|c_{1}-c\right\|+r_{L}\left(c_{1}-c\right) \\
& \leq 16 \varepsilon^{2}+8 \varepsilon M+\varepsilon \leq 16 \varepsilon+8 \varepsilon M+\varepsilon=(17+8 M) \varepsilon .
\end{aligned}
$$

Letting $\varepsilon \rightarrow 0$, we see that $\inf r_{L}(\operatorname{coinc}[f]-c) \leq 0$. Thus coinc $[f]$ is quasidense, and (a) holds.

Remark 4.5. An inspection of the above proof shows that we have, in fact, proved that if coinc $[f]$ is quasidense then coinc $[f]$ satisfies the stronger condition that, for all $c \in E \times E^{*}$, there exists $K_{c} \geq 0$ such that

$$
\inf \left\{r_{L}(a-c): a \in \operatorname{coinc}[f],\|a-c\| \leq K_{c}\right\} \leq 0 .
$$

It is clear from (4.1) that, for all $b, c \in E \times E^{*},\left(f_{c}-q_{L}\right)(b)=\left(f-q_{L}\right)(b+c)$. In light of this, the result of Lemma 4.6 below is very pleasing:

Lemma 4.6. Let $f \in \mathscr{P} \mathscr{C} \mathscr{L} \mathscr{S} \mathscr{C}\left(E \times E^{*}\right)$ and $f_{c}$ be as in (4.1). Then, for all $c \in E \times E^{*}$ and $b^{*} \in$ $E^{*} \times E^{* *},\left(f_{c}^{*}-q_{\widetilde{L}}\right)\left(b^{*}\right)=\left(f^{*}-q_{\widetilde{L}}\right)\left(b^{*}+L c\right)$.

Proof. From (4.1), the substitution $d=b+c$, (3.4) and (3.7),

$$
\begin{aligned}
\left(f_{c}^{*}-q_{\widetilde{L}}\right)\left(b^{*}\right) & =\sup _{b \in E \times E^{*}}\left[\left\langle b, b^{*}\right\rangle-\left(f-q_{L}\right)(b+c)-q_{L}(b)-q_{\widetilde{L}}\left(b^{*}\right)\right] \\
& =\sup _{d \in E \times E^{*}}\left[\left\langle d-c, b^{*}\right\rangle-\left(f-q_{L}\right)(d)-q_{L}(d-c)-q_{\widetilde{L}}\left(b^{*}\right)\right] \\
& =\sup _{d \in E \times E^{*}}\left[\left\langle d, b^{*}\right\rangle-f(d)+q_{L}(d)-q_{L}(d-c)-\left\langle c, b^{*}\right\rangle-q_{\widetilde{L}}\left(b^{*}\right)\right] \\
& =\sup _{d \in E \times E^{*}}\left[\left\langle d, b^{*}+L c\right\rangle-f(d)-q_{L}(c)-\left\langle c, b^{*}\right\rangle-q_{\widetilde{L}}\left(b^{*}\right)\right] \\
& =f^{*}\left(b^{*}+L c\right)-q_{\widetilde{L}}\left(b^{*}+L c\right) .
\end{aligned}
$$

This gives the required result.

Lemma 4.7. Let $f \in \mathscr{P} \mathscr{C}\left(E \times E^{*}\right), f \geq q_{L}$ on $E \times E^{*}$ and coinc $[f]$ be quasidense. Then $f^{*} \geq q_{\widetilde{L}}$ on $E^{*} \times E^{* *}$. 
Proof. Let $A:=\operatorname{coinc}[f]$. Let $c^{*} \in E^{*} \times E^{* *}$. Then, since $f=q_{L}$ on $A$,

$$
f^{*}\left(c^{*}\right)=\sup _{E \times E^{*}}\left[c^{*}-f\right] \geq \sup _{A}\left[c^{*}-f\right]=\sup _{A}\left[c^{*}-q_{L}\right] .
$$

Thus, from Definition 3.8 and Corollary 3.9, $f^{*}\left(c^{*}\right) \geq \Theta_{A}\left(c^{*}\right) \geq q_{\widetilde{L}}\left(c^{*}\right)$.

Theorem 4.8 (Dual condition for quasidensity). Let $f \in \mathscr{P} \mathscr{C} \mathscr{L} \mathscr{S} \mathscr{C}\left(E \times E^{*}\right)$ and $f \geq q_{L}$ on $E \times E^{*}$. Then coinc $[f]$ is quasidense $\Longleftrightarrow f^{*} \geq q_{\widetilde{L}}$ on $E^{*} \times E^{* *}$.

Proof. By virtue of Lemma 4.7, we only have to prove the implication ( $\Longleftarrow$ ). So assume that $f^{*} \geq q_{\widetilde{L}}$ on $E^{*} \times E^{* *}$. Let $c \in E \times E^{*}$. Let $f_{c} \in \mathscr{P} \mathscr{C} \mathscr{L} \mathscr{S} \mathscr{C}\left(E \times E^{*}\right)$ be as in (4.1). From Lemma 4.6, $f_{c}{ }^{*} \geq q_{\widetilde{L}} \geq$ $-\frac{1}{2}\|\cdot\|^{2}$ on $E^{*} \times E^{* *}$, thus $f_{c}{ }^{*}+\frac{1}{2}\|\cdot\|^{2} \geq 0$ on $E^{*} \times E^{* *}$. From (2.3), $\inf _{b \in E \times E^{*}}\left[f_{c}(b)+\frac{1}{2}\|b\|^{2}\right] \leq 0$. Thus, from Theorem 4.4 , coinc $[f]$ is quasidense, as required.

Definition 4.9. Let $f \in \mathscr{P} \mathscr{C}\left(E \times E^{*}\right)$. We define the function $f^{@}$ on $E \times E^{*}$ by $f^{@}:=f^{*} \circ L$. Explicitly, for all $a \in E \times E^{*}$,

$$
f^{@}(a):=\sup _{E \times E^{*}}[L a-f]=\sup _{b \in E \times E^{*}}[\langle b, L a\rangle-f(b)] .
$$

Lemma 4.10 will be used in Theorem 4.11, Lemma 6.14 and Theorem 6.15.

Lemma 4.10. Let $f, f^{@} \in \mathscr{P} \mathscr{C}\left(E \times E^{*}\right), f \geq q_{L}$ and $f^{@} \geq q_{L}$ on $E \times E^{*}$. Then $\operatorname{coinc}[f] \subset \operatorname{coinc}\left[f^{@}\right]$.

Proof. Let $a \in \operatorname{coinc}[f], b \in \operatorname{dom} f, \lambda, \mu>0$ and $\lambda+\mu=1$. Then

$$
\begin{aligned}
\lambda \mu q_{L}(a) & =\mu q_{L}(a)-\mu^{2} q_{L}(a)=\mu f(a)-\mu^{2} q_{L}(a) \\
& \geq f(\lambda b+\mu a)-\lambda f(b)-\mu^{2} q_{L}(a) \\
& \geq q_{L}(\lambda b+\mu a)-\lambda f(b)-\mu^{2} q_{L}(a) \\
& =\lambda^{2} q_{L}(b)+\lambda \mu\langle b, L a\rangle-\lambda f(b) .
\end{aligned}
$$

Dividing by $\lambda$ and letting $\lambda \rightarrow 0$, we see that $q_{L}(a) \geq\langle b, L a\rangle-f(b)$. If we now take the supremum over $b$ and use (4.9), we see that $q_{L}(a) \geq f^{@}(a)$. Consequently, $a \in \operatorname{coinc}\left[f^{@}\right]$.

The important thing about the next result is that $h$ is not required to be lower semicontinuous.

Theorem 4.11 (The theorem of the three functions). Let $h \in \mathscr{P} \mathscr{C}\left(E \times E^{*}\right)$,

$$
h \geq q_{L} \text { on } E \times E^{*} \text { and } h^{*} \geq q_{\widetilde{L}} \text { on } E^{*} \times E^{* *} .
$$

Then $h^{@} \geq q_{L}$ on $E \times E^{*}$ and coinc $\left[h^{@}\right]$ is closed and quasidense.

Proof. From (4.10), $\quad h^{@}=h^{*} \circ L \geq q_{\widetilde{L}} \circ L=q_{L}$ on $E \times E^{*}, \quad$ as required. From Theorem 2.1 or Theorem 2.3 with $k=q_{L}$, there exists $f \in \mathscr{P} \mathscr{C} \mathscr{L} \mathscr{S} \mathscr{C}\left(E \times E^{*}\right)$ such that $f \geq q_{L}$ on $E \times E^{*}$ and $f^{*}=h^{*} \geq q_{\widetilde{L}}$ on $E^{*} \times E^{* *}$, from which $f^{@}=h^{@} \geq q_{L}$ on $E \times E^{*}$. Thus Theorem 4.8 and Lemma 4.10 imply that coinc $[f]$ is quasidense and coinc $[f] \subset \operatorname{coinc}\left[f^{@}\right]$. Consequently, coinc $\left[f^{@}\right]$ is quasidense. Since $f^{@}=h^{@}$ on $E \times E^{*}$, coinc $\left[h^{@}\right]$ is quasidense also. Since $q_{L}$ is continuous and $h^{@}$ is lower semicontinuous, coinc $\left[h^{@}\right]$ is closed.

\section{THE COINCIDENCE SETS OF PARTIAL EPISUMS}

Let $E$ and $F$ be nonzero Banach spaces and $f, g \in \mathscr{P} \mathscr{C} \mathscr{L} \mathscr{S} \mathscr{C}(E \times F)$. Then we define the functions $f \oplus_{2} g$ and $f \oplus_{1} g$ by

$$
\left(f \oplus_{2} g\right)(x, y):=\inf _{\eta \in F}[f(x, y-\eta)+g(x, \eta)]
$$

and

$$
\left(f \oplus_{1} g\right)(x, y):=\inf _{\xi \in E}[f(x-\xi, y)+g(\xi, y)] .
$$


We substitute the symbol $\oplus_{2}^{e}$ for $\oplus_{2}$ and $\oplus_{1}^{e}$ for $\oplus_{1}$ if the infimum is exact, that is to say, can be replaced by a minimum. Lemma 5.1 below first appeared in Simons-Zălinescu [21, Section 4, pp. 8-10], and appeared subsequently in [15, Section 16, pp. 67-69]. It was later generalized in [16, Theorem 9, p. 882] and [17, Corollary 5.4, pp. 121-122]. We will be applying Lemmas 5.1 and 5.3 below with $F:=E^{*}$. We define the projection maps $\pi_{1}$ and $\pi_{2}$ by $\pi_{1}(x, y):=x$ and $\pi_{2}(x, y):=y((x, y) \in E \times F)$.

Lemma 5.1. Let $f, g \in \mathscr{P} \mathscr{C} \mathscr{L} \mathscr{S} \mathscr{C}(E \times F), f \oplus_{2} g \in \mathscr{P} \mathscr{C}(E \times F)$ and

$$
\bigcup_{\lambda>0} \lambda\left[\pi_{1} \operatorname{dom} f-\pi_{1} \operatorname{dom} g\right] \text { be a closed subspace of } E \text {. }
$$

Then $\left(f \oplus_{2} g\right)^{*}=f^{*} \oplus_{1}^{e} g^{*}$ on $E^{*} \times F^{*}$.

Theorem 5.2. Let $f, g \in \mathscr{P} \mathscr{C} \mathscr{L} \mathscr{S} \mathscr{C}\left(E \times E^{*}\right), f, g \geq q_{L}$ on $E \times E^{*}$,

$$
\bigcup_{\lambda>0} \lambda\left[\pi_{1} \operatorname{dom} f-\pi_{1} \operatorname{dom} g\right] \text { be a closed subspace of } E,
$$

and coinc $[f]$ and coinc $[g]$ be quasidense. Then $\left(f \oplus_{2} g\right)^{@} \geq q_{L}$ on $E \times E^{*}$, coinc $\left[\left(f \oplus_{2} g\right)^{@}\right]$ is closed and quasidense, and

$$
\left.\begin{array}{l}
\left(y, y^{*}\right) \in \operatorname{coinc}\left[\left(f \oplus_{2} g\right)^{@}\right] \Longleftrightarrow \\
\text { there exist } u^{*}, v^{*} \in E^{*} \text { such that } \\
\operatorname{inc}\left[f^{@}\right],\left(y, v^{*}\right) \in \operatorname{coinc}\left[g^{@}\right] \text { and } u^{*}+v^{*}=y^{*} .
\end{array}\right\}
$$

Proof. Let $h:=f \oplus_{2} g$. Since $f, g \geq q_{L}$ on $E \times E^{*}$, for all $\left(x, x^{*}\right) \in E \times E^{*}$,

$$
\begin{aligned}
h\left(x, x^{*}\right) & =\inf _{\xi^{*} \in E^{*}}\left[f\left(x, x^{*}-\xi^{*}\right)+g\left(x, \xi^{*}\right)\right] \\
& \geq \inf _{\xi^{*} \in E^{*}}\left[q_{L}\left(x, x^{*}-\xi^{*}\right)+q_{L}\left(x, \xi^{*}\right)\right] \\
& =\inf _{\xi^{*} \in E^{*}}\left[\left\langle x, x^{*}-\xi^{*}\right\rangle+\left\langle x, \xi^{*}\right\rangle\right] \\
& =\left\langle x, x^{*}\right\rangle=q_{L}\left(x, x^{*}\right) .
\end{aligned}
$$

From (5.2), $\pi_{1} \operatorname{dom} f \cap \pi_{1} \operatorname{dom} g \neq \emptyset$, and so there exist $x_{0} \in E, y_{0}^{*} \in E^{*}$ and $z_{0}^{*} \in E^{*}$ such that $\left(x_{0}, y_{0}^{*}\right) \in$ $\operatorname{dom} f$ and $\left(x_{0}, z_{0}^{*}\right) \in \operatorname{dom} g$. It now follows from (5.1) that

$$
\left(f \oplus_{2} g\right)\left(x_{0}, y_{0}^{*}+z_{0}^{*}\right) \leq f\left(x_{0}, y_{0}^{*}\right)+g\left(x_{0}, z_{0}^{*}\right)<\infty .
$$

To sum up:

$$
h \in \mathscr{P} \mathscr{C}\left(E \times E^{*}\right) \text { and } h \geq q_{L} \text { on } E \times E^{*} .
$$

Note that we do not assert in (5.4) that $h \in \mathscr{P} \mathscr{C} \mathscr{L} \mathscr{S} \mathscr{C}\left(E \times E^{*}\right)$. Since coinc $[f]$ and coinc $[g]$ are quasidense, Lemma 4.7 implies that

$$
f^{*} \geq q_{\widetilde{L}} \text { on } E^{*} \times E^{* *} \quad \text { and } \quad g^{*} \geq q_{\widetilde{L}} \text { on } E^{*} \times E^{* *},
$$

from which

$$
f^{@} \geq q_{L} \text { on } E \times E^{*} \quad \text { and } \quad g^{@} \geq q_{L} \text { on } E \times E^{*} .
$$

From Lemma 5.1 and (5.5), for all $\left(y^{*}, y^{* *}\right) \in E^{*} \times E^{* *}$,

$$
\left.\begin{array}{c}
h^{*}\left(y^{*}, y^{* *}\right)=\min _{z^{*} \in E^{*}}\left[f^{*}\left(y^{*}-z^{*}, y^{* *}\right)+g^{*}\left(z^{*}, y^{* *}\right)\right] \\
\geq \inf _{z^{*} \in E^{*}}\left[\left\langle y^{*}-z^{*}, y^{* *}\right\rangle+\left\langle z^{*}, y^{* *}\right\rangle\right]=\left\langle y^{*}, y^{* *}\right\rangle=q_{\widetilde{L}}\left(y^{*}, y^{* *}\right) .
\end{array}\right\}
$$

Thus $h^{*} \geq q_{\widetilde{L}}$ on $E^{*} \times E^{* *}$, and so (5.4) and Theorem 4.11 imply that $h^{@} \geq q_{L}$ on $E \times E^{*}$ and coinc $\left[h^{@}\right]$ is closed and quasidense, as required.

We now establish (5.3). If $\left(y, y^{*}\right) \in E \times E^{*}$ and we use (5.6) and specialize (5.7) to the case when $y^{* *}=\widehat{y}$, we obtain

$$
\left.\begin{array}{c}
h^{@}\left(y, y^{*}\right)=h^{*}\left(y^{*}, \widehat{y}\right)=\min _{z^{*} \in E^{*}}\left[f^{@}\left(y, y^{*}-z^{*}\right)+g^{@}\left(y, z^{*}\right)\right] \\
\geq \inf _{z^{*} \in E^{*}}\left[\left\langle y, y^{*}-z^{*}\right\rangle+\left\langle y, z^{*}\right\rangle\right]=\left\langle y, y^{*}\right\rangle=q_{L}\left(y, y^{*}\right) .
\end{array}\right\}
$$


If $\left(y, y^{*}\right) \in \operatorname{coinc}\left[h^{@}\right]$, then this provides $v^{*} \in E^{*}$ such that

$$
f^{@}\left(y, y^{*}-v^{*}\right)+g^{@}\left(y, v^{*}\right)=\left\langle y, y^{*}-v^{*}\right\rangle+\left\langle y, v^{*}\right\rangle .
$$

Let $u^{*}:=y^{*}-v^{*}$. Then $u^{*}+v^{*}=y^{*}$ and $f^{@}\left(y, u^{*}\right)+g^{@}\left(y, v^{*}\right)=\left\langle y, u^{*}\right\rangle+\left\langle y, v^{*}\right\rangle$. From (5.6), $\left(y, u^{*}\right) \in$ $\operatorname{coinc}\left[f^{@}\right]$ and $\left(y, v^{*}\right) \in \operatorname{coinc}\left[g^{\circledR}\right]$. This completes the proof of the implication $(\Longrightarrow)$ of (5.3). If, conversely, there exist $u^{*}, v^{*} \in E^{*}$ such that $\left(y, u^{*}\right) \in \operatorname{coinc}\left[f^{@}\right],\left(y, v^{*}\right) \in \operatorname{coinc}\left[g^{@}\right]$ and $u^{*}+v^{*}=y^{*}$ then, from (5.8),

$$
h^{@}\left(y, y^{*}\right) \leq f^{@}\left(y, u^{*}\right)+g^{@}\left(y, v^{*}\right)=\left\langle y, u^{*}\right\rangle+\left\langle y, v^{*}\right\rangle=\left\langle y, y^{*}\right\rangle .
$$

It now follows from (5.8) that $\left(y, y^{*}\right) \in \operatorname{coinc}\left[h^{@}\right]$. This completes the proof of the implication $(\Longleftarrow)$ of (5.3), and thus the proof of Theorem 5.2.

By interchanging the roles of $\oplus_{2}$ and $\oplus_{1}$ in the statement of Lemma 5.1, we can prove the following result:

Lemma 5.3. Let $f, g \in \mathscr{P} \mathscr{C} \mathscr{L} \mathscr{S} \mathscr{C}(E \times F), f \oplus_{1} g \in \mathscr{P} \mathscr{C}(E \times F)$ and

$$
\bigcup_{\lambda>0} \lambda\left[\pi_{2} \operatorname{dom} f-\pi_{2} \operatorname{dom} g\right] \text { be a closed subspace of } F \text {. }
$$

Then $\left(f \oplus_{1} g\right)^{*}=f^{*} \oplus_{2}^{e} g^{*}$ on $E^{*} \times F^{*}$.

Theorem 5.4. Let $f, g \in \mathscr{P} \mathscr{C} \mathscr{L} \mathscr{S} \mathscr{C}\left(E \times E^{*}\right), f, g \geq q_{L}$ on $E \times E^{*}$,

$$
\bigcup_{\lambda>0} \lambda\left[\pi_{2} \operatorname{dom} f-\pi_{2} \operatorname{dom} g\right] \text { be a closed subspace of } E^{*},
$$

and coinc $[f]$ and coinc $[g]$ be quasidense. Then $\left(f \oplus_{1} g\right)^{@} \geq q_{L}$ on $E \times E^{*}$, coinc $\left[\left(f \oplus_{1} g\right)^{@}\right]$ is closed and quasidense and,

$$
\left.\begin{array}{l}
\left(y, y^{*}\right) \in \operatorname{coinc}\left[\left(f \oplus_{1} g\right)^{@}\right] \Longleftrightarrow \\
\text { there exist } u^{* *}, v^{* *} \in E^{* *} \text { such that } \\
\operatorname{oinc}\left[f^{*}\right],\left(y^{*}, v^{* *}\right) \in \operatorname{dcoinc}\left[g^{*}\right] \text { and } u^{* *}+v^{* *}=\widehat{y} .
\end{array}\right\}
$$

Proof. Let $h:=f \oplus_{1} g$. By interchanging the variables in the proofs already given of (5.4) and (5.5) in Theorem 5.2, we can prove that,

$$
h \in \mathscr{P} \mathscr{C}\left(E \times E^{*}\right) \text { and } h \geq q_{L} \text { on } E \times E^{*}
$$

and

$$
f^{*} \geq q_{\widetilde{L}} \text { on } E^{*} \times E^{* *} \quad \text { and } \quad g^{*} \geq q_{\widetilde{L}} \text { on } E^{*} \times E^{* *} .
$$

From Lemma 5.3 and (5.12), for all $\left(y^{*}, y^{* *}\right) \in E^{*} \times E^{* *}$,

$$
\left.\begin{array}{c}
h^{*}\left(y^{*}, y^{* *}\right)=\min _{z^{* *} \in E^{* *}}\left[f^{*}\left(y^{*}, y^{* *}-z^{* *}\right)+g^{*}\left(y^{*}, z^{* *}\right)\right. \\
\geq \inf _{z^{* *} \in E^{* *}}\left[\left\langle y^{*}, y^{* *}-z^{* *}\right\rangle+\left\langle y^{*}, z^{* *}\right\rangle\right]=\left\langle y^{*}, y^{* *}\right\rangle=q_{\widetilde{L}}\left(y^{*}, y^{* *}\right) .
\end{array}\right\}
$$

Thus $h^{*} \geq q_{\widetilde{L}}$ on $E^{*} \times E^{* *}$, and so (5.11) and Theorem 4.11 imply that $h^{@} \geq q_{L}$ on $E \times E^{*}$ and coinc $\left[h^{@}\right]$ is closed and quasidense, as required. If we now let $\left(y, y^{*}\right) \in E \times E^{*}$ and specialize (5.13) to the case when $y^{* *}=\widehat{y}$, we obtain

$$
\left.\begin{array}{c}
h^{@}\left(y, y^{*}\right)=\min _{z^{* *} \in E^{* *}}\left[f^{*}\left(y^{*}, \widehat{y}-z^{* *}\right)+g^{*}\left(y^{*}, z^{* *}\right)\right] \\
\geq \inf _{z^{* *} \in E^{* *}}\left[\left\langle y^{*}, \widehat{y}-z^{* *}\right\rangle+\left\langle y^{*}, z^{* *}\right\rangle\right]=\left\langle y, y^{*}\right\rangle=q_{L}\left(y, y^{*}\right) .
\end{array}\right\}
$$

We now establish (5.10). If $\left(y, y^{*}\right) \in \operatorname{coinc}\left[h^{@}\right]$ then (5.14) provides $v^{* *} \in E^{* *}$ such that

$$
f^{*}\left(y^{*}, \widehat{y}-v^{* *}\right)+g^{*}\left(y^{*}, v^{* *}\right)=\left\langle y^{*}, \widehat{y}-v^{* *}\right\rangle+\left\langle y^{*}, v^{* *}\right\rangle .
$$

Let $u^{* *}:=\widehat{y}-v^{* *}$. Then we have $u^{* *}+v^{* *}=\widehat{y}$ and $f^{*}\left(y^{*}, u^{* *}\right)+g^{*}\left(y^{*}, v^{* *}\right)=\left\langle y^{*}, u^{* *}\right\rangle+\left\langle y^{*}, v^{* *}\right\rangle$. From (5.12), $\left(y^{*}, u^{* *}\right) \in \operatorname{dcoinc}\left[f^{*}\right]$ and $\left(y^{*}, v^{* *}\right) \in \operatorname{dcoinc}\left[g^{*}\right]$. This completes the proof of the implication $(\Longrightarrow)$ 
of (5.10). If, conversely, there exist $u^{* *}, v^{* *} \in E^{* *}$ such that $\left(y^{*}, u^{* *}\right) \in \operatorname{dcoinc}\left[f^{*}\right],\left(y^{*}, v^{* *}\right) \in \operatorname{dcoinc}\left[g^{*}\right]$ and $u^{* *}+v^{* *}=\widehat{y}$ then, from (5.14),

$$
h^{@}\left(y, y^{*}\right) \leq f^{*}\left(y^{*}, u^{* *}\right)+g^{*}\left(y^{*}, v^{* *}\right)=\left\langle y^{*}, u^{* *}\right\rangle+\left\langle y^{*}, v^{* *}\right\rangle=\left\langle y^{*}, \widehat{y}\right\rangle=\left\langle y, y^{*}\right\rangle .
$$

It now follows from (5.14) that $\left(y, y^{*}\right) \in \operatorname{coinc}\left[h^{@}\right]$. This completes the proof of the implication $(\Longleftarrow)$ of (5.10), and thus the proof of Theorem 5.4.

\section{Monotone Sets And MUltifunctions}

Let $\emptyset \neq A \subset E \times E^{*}$. It is easy to see that

$A$ is monotone if, and only if, for all $a, b \in A, q_{L}(a-b) \geq 0$

if, and only if, $L(A)$ is a monotone subset of $E^{*} \times E^{* *}$.

Theorem 6.1 (Quasidensity and maximality). Let A be a closed, quasidense monotone subset of $E \times E^{*}$. Then A is maximally monotone.

Proof. Let $c \in E \times E^{*}$ and $A \cup\{c\}$ be monotone. Let $\varepsilon>0$, and choose $a \in A$ so that $r_{L}(a-c)<\varepsilon$. Since $q_{L}(a-c) \geq 0$, it follows that

$$
\frac{1}{2}\|a-c\|^{2} \leq \frac{1}{2}\|a-c\|^{2}+q_{L}(a-c)=r_{L}(a-c)<\varepsilon .
$$

Letting $\varepsilon \rightarrow 0$ and using the fact that $A$ is closed, one has $c \in A$.

The following important property of coincidence sets was first proved in Burachik-Svaiter, [4, Theorem 3.1, pp. 2381-2382] and Penot, [11, Proposition 4(h) $\Longrightarrow(a)$, pp. 860-861]. Here, we give a short proof using the criterion for monotonicity that appeared in (6.1).

Lemma 6.2. Let $f \in \mathscr{P} \mathscr{C}\left(E \times E^{*}\right)$ and $f \geq q_{L}$ on $E \times E^{*}$. Then coinc $[f]$ is monotone.

Proof. Let $a, b \in \operatorname{coinc}[f]$. Then

$$
\begin{aligned}
\frac{1}{4} q_{L}(a-b) & =\frac{1}{2} q_{L}(a)+\frac{1}{2} q_{L}(b)-\frac{1}{4} q_{L}(a+b) \\
& =\frac{1}{2} f(a)+\frac{1}{2} f(b)-\frac{1}{4} q_{L}(a+b) \\
& \geq f\left(\frac{1}{2}(a+b)\right)-q_{L}\left(\frac{1}{2}(a+b)\right) \geq 0 .
\end{aligned}
$$

This establishes (6.1) and completes the proof of Lemma 6.2.

In order to simplify some notation in the sequel, if $S: E \rightrightarrows E^{*}$, we will say that $S$ is closed if its graph, $G(S)$, is closed in $E \times E^{*}$, and we will say that $S$ is quasidense if $G(S)$ is quasidense in $E \times E^{*}$.

Our analysis depends on the following definition:

Definition 6.3 (The definition of $\theta_{S}$ ). Let $S: E \rightrightarrows E^{*}$ be a monotone multifunction and $G(S) \neq \emptyset$. We define the function $\theta_{S} \in \mathscr{P} \mathscr{C} \mathscr{L} \mathscr{S} \mathscr{C}\left(E^{*} \times E^{* *}\right)$ by $\theta_{S}:=\Theta_{G(S)}$. (See Definition 3.8.) Explicitly:

$$
\text { for all } c^{*} \in E^{*} \times E^{* *}, \quad \theta_{S}\left(c^{*}\right):=\sup _{G(S)}\left[c^{*}-q_{L}\right] .
$$

In longhand, for all $\left(y^{*}, y^{* *}\right) \in E^{*} \times E^{* *}$

$$
\theta_{S}\left(y^{*}, y^{* *}\right):=\sup _{\left(s, s^{*}\right) \in G(S)}\left[\left\langle s, y^{*}\right\rangle+\left\langle s^{*}, y^{* *}\right\rangle-\left\langle s, s^{*}\right\rangle\right] .
$$

We now show how $\theta_{S}$ determines the Fitzpatrick function, $\varphi_{S}$, that acts on $E \times E^{*}$ (rather than on $\left.E^{*} \times E^{* *}\right)$. 
Definition 6.4 (The definition of $\varphi_{S}$ ). Let $S: E \rightrightarrows E^{*}$ be a monotone multifunction and $G(S) \neq \emptyset$. We define the function $\varphi_{S} \in \mathscr{P} \mathscr{C} \mathscr{L} \mathscr{S} \mathscr{C}\left(E \times E^{*}\right)$ by

$$
\varphi_{S}=\theta_{S} \circ L
$$

Explicitly,

$$
\text { for all } b \in E \times E^{*}, \quad \begin{aligned}
\varphi_{S}(b) & :=\sup _{G(S)}\left[L b-q_{L}\right] \\
& =q_{L}(b)-\inf q_{L}(G(S)-b) .
\end{aligned}
$$

In longhand, for all $\left(x, x^{*}\right) \in E \times E^{*}$,

$$
\varphi_{S}\left(x, x^{*}\right):=\sup _{\left(s, s^{*}\right) \in G(S)}\left[\left\langle s, x^{*}\right\rangle+\left\langle x, s^{*}\right\rangle-\left\langle s, s^{*}\right\rangle\right] .
$$

Remark 6.5. The Fitzpatrick function was originally introduced in the Banach space setting in [5, (1988)], but lay dormant until it was rediscovered by Martínez-Legaz and Théra in [10, (2001)]. It had been previously considered in the finite-dimensional setting by Krylov in [9, (1982)]. The generalization of the Fitzpatrick function to Banach SN spaces can be found in [18, Definition 6.2, p. 1029].

Lemma 6.6. Let $S: E \rightrightarrows E^{*}$ be maximally monotone. Then:

$$
\varphi_{S} \in \mathscr{P} \mathscr{C} \mathscr{L} \mathscr{S} \mathscr{C}\left(E \times E^{*}\right), \quad \varphi_{S} \geq q_{L} \text { on } E \times E^{*} \quad \text { and } \quad \operatorname{coinc}\left[\varphi_{S}\right]=G(S) .
$$

Proof. If $b \in E \times E^{*}$ and $\varphi_{S}(b) \leq q_{L}(b)$ then (6.7) gives inf $q_{L}(G(S)-b) \geq 0$. From the maximality, $b \in G(S)$ and so we derive from the monotonicity that inf $q_{L}(G(S)-b)=0$, from which $\varphi_{S}(b)=q_{L}(b)$. Since $\varphi_{S}$ is obviously convex and lower semicontinuous, this completes the proof of (6.9).

We now come to the " $\varphi_{S}{ }^{*}$ criterion" for a maximally monotone set to be quasidense.

Theorem 6.7. Let $S: E \rightrightarrows E^{*}$ be maximally monotone. Then:

$$
S \text { is quasidense } \Longleftrightarrow \varphi_{S}{ }^{*} \geq q_{\widetilde{L}} \text { on } E^{*} \times E^{* *} .
$$

Proof. This is immediate from (6.9) and Theorem 4.8.

Corollary 6.8 (First partial converse to Theorem 6.1). Let $S: E \rightrightarrows E^{*}$ be maximally monotone and surjective. Then $S$ is quasidense.

Proof. Suppose that $\left(y^{*}, y^{* *}\right) \in E^{*} \times E^{* *}$. Let $x \in S^{-1} y^{*}$. Then, from (6.9),

$$
\begin{aligned}
\varphi_{S}^{*}\left(y^{*}, y^{* *}\right) & \geq\left\langle x, y^{*}\right\rangle+\left\langle y^{*}, y^{* *}\right\rangle-\varphi_{S}\left(x, y^{*}\right) \\
& =\left\langle x, y^{*}\right\rangle+\left\langle y^{*}, y^{* *}\right\rangle-\left\langle x, y^{*}\right\rangle \\
& =\left\langle y^{*}, y^{* *}\right\rangle=q_{\widetilde{L}}\left(y^{*}, y^{* *}\right) .
\end{aligned}
$$

It now follows from (6.10) that $S$ is quasidense.

Remark 6.9. Once one knows the (highly nontrivial) result that a maximally monotone multifunction is quasidense if, and only if, it is of type (FP), or locally maximally monotone, see [19, Theorem 10.3, p. 21], then Corollary 6.8 follows from Fitzpatrick-Phelps, [6, Theorem 3.7, pp. 67-68].

In Theorem 6.12, we will give the " $\theta_{S}$ criterion" for a maximally monotone set to be quasidense. We start with a preliminary lemma of independent interest, which will be used in Corollary 11.7. Lemma 6.11 raises the following problem:

Problem 6.10. Is there a maximally monotone multifunction $S: E \rightrightarrows E^{*}$ such that $\varphi_{S}{ }^{*} \neq \theta_{S}$ ? 
Lemma 6.11. Let $S: E \rightrightarrows E^{*}$ be maximally monotone. Then:

$$
\varphi_{S}{ }^{*} \geq \theta_{S} \text { on } E^{*} \times E^{* *} \text {. }
$$

If, further,

$$
\operatorname{dom} \varphi_{S} \subset G(S)
$$

then

$$
\varphi_{S}^{*}=\theta_{S} \text { on } E^{*} \times E^{* *} \text {. }
$$

Proof. Let $c^{*} \in E^{*} \times E^{* *}$. From (6.9) and (6.3),

$$
\varphi_{S}{ }^{*}\left(c^{*}\right)=\sup _{E \times E^{*}}\left[c^{*}-\varphi_{S}\right] \geq \sup _{G(S)}\left[c^{*}-\varphi_{S}\right]=\sup _{G(S)}\left[c^{*}-q_{L}\right]=\theta_{S}\left(c^{*}\right),
$$

which gives (6.11). Now suppose that (6.12) is satisfied. If $b \in E \times E^{*} \backslash \operatorname{dom} \varphi_{S}$ then $\left\langle b, c^{*}\right\rangle-\varphi_{S}(b)=$ $-\infty \leq \theta_{S}\left(c^{*}\right)$. If, on the other hand, $b \in \operatorname{dom} \varphi_{S}$ then (6.12) implies that $b \in G(S)$, and so (6.9) gives $\varphi_{S}(b)=q_{L}(b)$. Thus, using (6.3), $\left\langle b, c^{*}\right\rangle-\varphi_{S}(b)=\left\langle b, c^{*}\right\rangle-q_{L}(b) \leq \theta_{S}\left(c^{*}\right)$. Combining these two observations, we see that,

$$
\text { for all } b \in E \times E^{*}, \quad\left\langle b, c^{*}\right\rangle-\varphi_{S}(b) \leq \theta_{S}\left(c^{*}\right) .
$$

Taking the supremum over $b \in E \times E^{*}, \varphi_{S}{ }^{*}\left(c^{*}\right) \leq \theta_{S}\left(c^{*}\right)$. Thus $\varphi_{S}{ }^{*} \leq \theta_{S}$ on $E^{*} \times E^{* *}$, and (6.13) follows from (6.11).

Theorem 6.12. Let $S: E \rightrightarrows E^{*}$ be maximally monotone. Then:

$$
S \text { is quasidense } \Longleftrightarrow \theta_{S} \geq q_{\widetilde{L}} \text { on } E^{*} \times E^{* *} \text {. }
$$

Proof. If $S$ is quasidense then $G(S)$ is a quasidense subset of $E \times E^{*}$ and so, from Corollary 3.9, $\theta_{S}=$ $\Theta_{G(S)} \geq q_{\widetilde{L}}$ on $E^{*} \times E^{* *}$. If, conversely, $\theta_{S} \geq q_{\widetilde{L}}$ on $E^{*} \times E^{* *}$ then, from (6.11), $\varphi_{S}{ }^{*} \geq q_{\widetilde{L}}$ on $E^{*} \times E^{* *}$, and it follows from Theorem 6.7 that $S$ is quasidense.

Corollary 6.13 (Second partial converse to Theorem 6.1). Let $E$ be reflexive and $S: E \rightrightarrows E^{*}$ be maximally monotone. Then $S$ is quasidense.

Proof. Suppose that $\left(y^{*}, y^{* *}\right) \in E^{*} \times E^{* *}$. Choose $y \in E$ such that $\widehat{y}=y^{* *}$. Then $\left(y^{*}, y^{* *}\right)=\left(y^{*}, \widehat{y}\right)=$ $L\left(y, y^{*}\right)$ and so, from (6.5) and (6.9),

$$
\theta_{S}\left(y^{*}, y^{* *}\right)=\theta_{S} \circ L\left(y, y^{*}\right)=\varphi_{S}\left(y, y^{*}\right) \geq q_{L}\left(y, y^{*}\right)=q_{\widetilde{L}}\left(y^{*}, y^{* *}\right) .
$$

It now follows from Theorem 6.12 that $S$ is quasidense.

We end this section by giving a result in Theorem 6.15 that will be used in our discussion of the Fitzpatrick extension in Section 8. We start with a preliminary lemma.

Lemma 6.14. Let $S: E \rightrightarrows E^{*}$ be maximally monotone. Then:

$$
\begin{gathered}
\varphi_{S}{ }^{@} \geq \varphi_{S} \geq q_{L} \text { on } E \times E^{*} \text { and } \operatorname{coinc}\left[\varphi_{S}{ }^{@}\right]=G(S) . \\
\theta_{S}{ }^{@} \geq \varphi_{S}{ }^{*} \geq \theta_{S} \text { on } E^{*} \times E^{* *} .
\end{gathered}
$$

Proof. It follows by composing (6.11) with $L$ and using Definition 4.9 and (6.5) that $\varphi_{S}{ }^{\circledR} \geq \varphi_{S}$ on $E \times E^{*}$. Furthermore, (6.9) implies that $\varphi_{S} \geq q_{L}$ on $E \times E^{*}$ and $G(S)=\operatorname{coinc}\left[\varphi_{S}\right] \supset \operatorname{coinc}\left[\varphi_{S}{ }^{\circledR}\right]$. Lemma 4.10 implies that coinc $\left[\varphi_{S}\right] \subset \operatorname{coinc}\left[\varphi_{S}{ }^{\circledR}\right]$, which completes the proof of (6.15).

For all $c^{*} \in E^{*} \times E^{* *}, \theta_{S}{ }^{@}\left(c^{*}\right)=\sup _{E^{*} \times E^{* *}}\left[\widetilde{L} c^{*}-\theta_{S}\right]$. Thus, from (6.5),

$$
\begin{aligned}
\theta_{S}{ }^{@}\left(c^{*}\right) & \geq \sup _{b \in E \times E^{*}}\left[\left\langle L b, \widetilde{L} c^{*}\right\rangle-\theta_{S}(L b)\right] \\
& =\sup _{b \in E \times E^{*}}\left[\left\langle b, c^{*}\right\rangle-\varphi_{S}(b)\right]=\varphi_{S}{ }^{*}\left(c^{*}\right),
\end{aligned}
$$

which gives the first inequality in (6.16), and the second inequality in (6.16) has already been established in (6.11). 
Theorem 6.15. Let $S: E \rightrightarrows E^{*}$ be maximally monotone and quasidense. Then $\operatorname{dcoinc}\left[\theta_{S}\right]=\operatorname{dcoinc}\left[\varphi_{S}{ }^{*}\right]$ $=\operatorname{dcoinc}\left[\theta_{S}{ }^{\circledR}\right]$.

Proof. From (6.16) and (6.14), $\quad \theta_{S}{ }^{@} \geq \varphi_{S}{ }^{*} \geq \theta_{S} \geq q_{\widetilde{L}}$ on $E^{*} \times E^{* *}$. It follows that $\operatorname{dcoinc}\left[\theta_{S}{ }^{\circledR}\right] \subset$ $\operatorname{dcoinc}\left[\varphi_{S}{ }^{*}\right] \subset \operatorname{dcoinc}\left[\theta_{S}\right]$. However, if we apply Lemma 4.10 (to $E^{*} \times E^{* *}$ instead of $E \times E^{*}$ ), we see that dcoinc $\left[\theta_{S}\right] \subset \operatorname{dcoinc}\left[\theta_{S}{ }^{\circledR}\right]$. This gives the desired result.

Problem 6.16. Theorem 6.15 leads to the question: if $S$ is maximally monotone and $\theta_{S}{ }^{@} \geq q_{\widetilde{L}}$ on $E^{*} \times E^{* *}$ then is $S$ necessarily quasidense?

\section{SUM THEOREM WITH DOMAIN CONSTRAINTS}

Notation 7.1. Let $S: E \rightrightarrows E^{*}$. In what follows, we write

$$
D(S):=\{x \in E: S x \neq \emptyset\}=\pi_{1} G(S) \text { and } R(S):=\bigcup_{x \in E} S x=\pi_{2} G(S) .
$$

We will use the following computational rules in the sequel:

Lemma 7.2. Let $S: E \rightrightarrows E^{*}$ be closed, quasidense and monotone. Then

$$
D(S) \subset \pi_{1} \operatorname{dom} \varphi_{S} \quad \text { and } \quad R(S) \subset \pi_{2} \operatorname{dom} \varphi_{S} .
$$

Proof. This is immediate from Theorem 6.1 and (6.9).

Theorem 7.3 (Sum theorem with domain constraints). Let $S, T: E \rightrightarrows E^{*}$ be closed, quasidense and monotone. Then $(\mathrm{a}) \Longrightarrow(\mathrm{b}) \Longrightarrow(\mathrm{c}) \Longrightarrow(\mathrm{d})$ :

(a) $D(S) \cap \operatorname{int} D(T) \neq \emptyset$ or $\operatorname{int} D(S) \cap D(T) \neq \emptyset$.

(b) $\bigcup_{\lambda>0} \lambda[D(S)-D(T)]=E$.

(c) $\cup_{\lambda>0} \lambda\left[\pi_{1} \operatorname{dom} \varphi_{S}-\pi_{1} \operatorname{dom} \varphi_{T}\right]$ is a closed subspace of $E$.

(d) $S+T$ is closed, quasidense and monotone.

Proof. It is immediate from (7.1) that $(\mathrm{a}) \Longrightarrow(\mathrm{b}) \Longrightarrow(\mathrm{c})$. Now suppose that (c) is satisfied. From Theorem 6.1, $S$ and $T$ are maximally monotone, and so (6.9) and (6.15) imply that the functions $\varphi_{S}, \varphi_{T} \in$ $\mathscr{P} \mathscr{C} \mathscr{L} \mathscr{S} \mathscr{C}\left(E \times E^{*}\right), \varphi_{S}, \varphi_{T} \geq q_{L}$ on $E \times E^{*}, \operatorname{coinc}\left[\varphi_{S}\right]=\operatorname{coinc}\left[\varphi_{S}{ }^{\circledR}\right]=G(S) \quad$ and $\operatorname{coinc}\left[\varphi_{T}\right]=$ $\operatorname{coinc}\left[\varphi_{T}{ }^{@}\right]=G(T)$, and we can apply Theorem 5.2 with $f:=\varphi_{S}$ and $g:=\varphi_{T}$.

Thus $\left(\varphi_{S} \oplus_{2} \varphi_{T}\right)^{@} \geq q_{L}$ on $E \times E^{*}, \operatorname{coinc}\left[\left(\varphi_{S} \oplus_{2} \varphi_{T}\right)^{@}\right]$ is closed and quasidense, and $\left(y, y^{*}\right) \in$ $\operatorname{coinc}\left[\left(\varphi_{S} \oplus_{2} \varphi_{T}\right)^{@}\right]$ if, and only if, there exist $u^{*}, v^{*} \in E^{*}$ such that $\left(y, u^{*}\right) \in G(S),\left(y, v^{*}\right) \in G(T)$ and $u^{*}+v^{*}=y^{*}$. This is exactly equivalent to the statement that $\left(y, y^{*}\right) \in G(S+T)$. Finally, it is obvious that $S+T$ is monotone.

Remark 7.4. Theorem 7.3 above has applications to the classification of maximally monotone multifunctions. See [19, Theorems 7.2 and 8.1]. Theorem 7.3 can also be deduced from Voisei-Zălinescu [22, Corollary 3.5, p. 1024].

\section{THE FITZPATRICK EXTENSION}

Definition 8.1 (The Fitzpatrick extension). Let $S: E \rightrightarrows E^{*}$ be a closed quasidense monotone multifunction. We now introduce the Fitzpatrick extension, $S^{\mathbb{F}}: E^{*} \rightrightarrows E^{* *}$, of $S$. From Theorem 6.1 and (6.9), $\operatorname{coinc}\left[\varphi_{S}\right]=G(S)$, and so we see from Theorem 4.8 that $\varphi_{S}{ }^{*} \geq q_{\widetilde{L}}$ on $E^{*} \times E^{* *}$. Using our current notation, the multifunction $S^{\mathbb{F}}$ was defined in [19, Definition 5.1] by

$$
G\left(S^{\mathbb{F}}\right):=\operatorname{dcoinc}\left[\varphi_{S}{ }^{*}\right] .
$$

(There is a more abstract version of this in [18, Definition 8.5, p. 1037].) From Theorem 6.15, we can also write

$$
G\left(S^{\mathbb{F}}\right)=\operatorname{dcoinc}\left[\theta_{S}\right]=\operatorname{dcoinc}\left[\theta_{S}^{@}\right] .
$$


The word extension is justified by the fact that $L(a) \in G\left(S^{\mathbb{F}}\right) \Longleftrightarrow a \in G(S)$. Indeed, from (8.2), (6.5) and (6.9),

$$
\left.\begin{array}{c}
L(a) \in G\left(S^{\mathbb{F}}\right) \Longleftrightarrow \theta_{S}(L(a))=q_{\widetilde{L}}(L(a)) \\
\Longleftrightarrow \varphi_{S}(a)=q_{L}(a) \Longleftrightarrow a \in G(S) .
\end{array}\right\}
$$

Theorem 8.2. Let $S: E \rightrightarrows E^{*}$ be closed, quasidense and monotone. Then $S^{\mathbb{F}}$ is maximally monotone.

Proof. From Lemma 6.2 (applied to the function $\varphi_{S}{ }^{*}$ on $\left.E^{*} \times E^{* *}\right), S^{\mathbb{F}}$ is monotone. Now let $c^{*} \in$ $E^{*} \times E^{* *}$ and, for all $a^{*} \in G\left(S^{\mathbb{F}}\right), q_{\widetilde{L}}\left(c^{*}-a^{*}\right) \geq 0$. From (8.3), for all $a \in G(S), q_{\widetilde{L}}\left(c^{*}-L(a)\right) \geq 0$. Now (3.7) gives $q_{\widetilde{L}}\left(c^{*}-L(a)\right)=q_{\widetilde{L}}\left(c^{*}\right)-\left\langle a, c^{*}\right\rangle+q_{L}(a)$ and so, for all $a \in G(S), q_{\widetilde{L}}\left(c^{*}\right) \geq\left\langle a, c^{*}\right\rangle-q_{L}(a)$. Taking the supremum over $a$ and using (6.3), $q_{\widetilde{L}}\left(c^{*}\right) \geq \theta_{S}\left(c^{*}\right)$. From Theorem 6.12, $\theta_{S}\left(c^{*}\right)=q_{\widetilde{L}}\left(c^{*}\right)$, and so $c^{*} \in \operatorname{dcoinc}\left[\theta_{S}\right]$. Thus, from (8.2), $c^{*} \in G\left(S^{\mathbb{F}}\right)$. This completes the proof of the maximal monotonicity of $S^{\mathbb{F}}$.

Remark 8.3. It is interesting to speculate (see [18, Problem 12.7, p. 1047]) whether $S^{\mathbb{F}}$ is actually quasidense. We shall see in Example 8.8, Theorems 11.4(b) and 12.3 that this is not generally the case. However, it is the case in one important situation. We observed in Example 3.2 that if $f: E \rightarrow]-\infty, \infty]$ is proper, convex and lower semicontinuous then $\partial f: E \rightrightarrows E^{*}$ is quasidense. However, it was shown in [19, Theorem 5.7] that $(\partial f)^{\mathbb{F}}=\partial\left(f^{*}\right)$, so the multifunction $(\partial f)^{\mathbb{F}}: E^{*} \rightrightarrows E^{* *}$ is quasidense.

Remark 8.4. It follows from (8.2) that $y^{* *} \in S^{\mathbb{F}}\left(y^{*}\right)$ exactly when $\left(y^{* *}, y^{*}\right)$ is in the Gossez extension of $G(S)$ (see [7, Lemma 2.1, p. 275]).

Our next result gives a situation in which we can obtain an explicit description of $S^{\mathbb{F}}$, as well as inverse of the operation $S \mapsto S^{\mathbb{F}}$. Theorem 8.5 is an extension to the nonlinear case of [2, Theorem 2.1, pp. 297-298]. It will be important in our construction of examples.

Theorem 8.5. Let $T: E^{*} \rightrightarrows E^{* *}$ and $R(T) \subset \widehat{E}$. Let $S=G^{-1} L^{-1} G(T)$, i.e., $S: E \rightrightarrows E^{*}$ is defined by $G(S)=L^{-1} G(T)$. Then:

(a) $G(T) \subset L(G(S))$. (The opposite inclusion is trivially true.)

(b) Suppose in addition that $T$ is maximally monotone. Then $S$ is maximally monotone.

(c) Suppose in addition that $T$ is maximally monotone and $D(T)=E^{*}$. Then $S$ is maximally monotone and quasidense, and $S^{\mathbb{F}}=T$. Put another way, for multifunctions like $T, G^{-1} L^{-1} G$ is the inverse of ${ }^{\mathbb{F}}$.

Proof. (a) Let $\left(y^{*}, y^{* *}\right) \in G(T)$. Since $R(T) \subset \widehat{E}$, there exists $y \in E$ such that $y^{* *}=\widehat{y}$. But then $\left(y^{*}, y^{* *}\right)=$ $L\left(y, y^{*}\right)$, and so $\left(y, y^{*}\right) \in L^{-1}\left\{\left(y^{*}, y^{* *}\right)\right\} \subset L^{-1} G(T)=G(S)$, from which $\left(y^{*}, y^{* *}\right)=L\left(y, y^{*}\right) \in L(G(S))$.

(b) Now let $b_{1}, b_{2} \in G(S)$. Then $L b_{1}, L b_{2} \in G(T)$, and so $q_{\widetilde{L}}\left(L b_{1}-L b_{2}\right) \geq 0$. Equivalently, $q_{L}\left(b_{1}-\right.$ $\left.b_{2}\right) \geq 0$. Thus $S$ is monotone. We now prove that $S$ is maximally monotone. To this end, let $c \in E \times E^{*}$ and $\inf q_{L}(G(S)-c) \geq 0$. Equivalently, $\inf q_{\widetilde{L}}(L(G(S))-L c) \geq 0$. From (a), $\inf q_{\widetilde{L}}(G(T)-L c) \geq 0$. The maximal monotonicity of $T$ now implies that $L c \in L(G(S))$. Since $L$ is injective, $c \in G(S)$. Thus $S$ is maximally monotone.

(c) Let $y^{*} \in E^{*}=D(T)$. Arguing as in (a), there exist $y^{* *} \in E^{* *}$ and $y \in E$ such that $L\left(y, y^{*}\right)=$ $\left(y^{*}, y^{* *}\right) \in L(G(S))$. Since $L$ is injective, $\left(y, y^{*}\right) \in G(S)$. Thus $R(S)=E^{*}$, and the quasidensity of $S$ follows from (b) and Corollary 6.8. (8.3) and (a) now imply that that $G\left(S^{\mathbb{F}}\right) \supset L(G(S)) \supset G(T)$, and the assumed maximal monotonicity of $T$ now gives $S^{\mathbb{F}}=T$, as required.

The following result appears in Phelps-Simons, [12, Corollary 2.6, p. 306]. We do not know the original source of the result. We almost certainly learned about it by personal communication with Robert Phelps. We give a proof for completeness.

Fact 8.6. Let $T: E \rightarrow E^{*}$ be monotone and linear. Then $T$ is maximally monotone. 
Proof. Let $\left(y, y^{*}\right) \in E \times E^{*}$ and, for all $x \in E,\left\langle x-y, T x-y^{*}\right\rangle \geq 0$. We first prove that, for all $z \in E$ and for all $\lambda \in \mathbb{R}$,

$$
\lambda\left\langle z, T y-y^{*}\right\rangle+\lambda^{2}\langle z, T z\rangle \geq 0 .
$$

To this end, let $z \in E$ and $\lambda \in \mathbb{R}$. By direct computation,

$$
\lambda\left\langle z, T y-y^{*}\right\rangle+\lambda^{2}\langle z, T z\rangle=\left\langle y+\lambda z-y, T(y+\lambda z)-y^{*}\right\rangle .
$$

(8.4) now follows from our assumption, with $x=y+\lambda z$. From (8.4), for all $z \in E$, the quadratic expression $\lambda \mapsto \lambda\left\langle z, T y-y^{*}\right\rangle+\lambda^{2}\langle z, T z\rangle$ attains a minimum at $\lambda=0$ so, from elementary calculus, for all $z \in E,\left\langle z, T y-y^{*}\right\rangle=0$. Consequently, $T y-y^{*}=0 \in E^{*}$. Thus $y^{*}=T y$. This completes the proof of the maximal monotonicity of $T$.

Theorem 8.7 will be applied in Example 8.8 and Theorem 11.4.

Theorem 8.7. Let $T: E^{*} \rightarrow E^{* *}$ be a monotone linear map and $R(T) \subset \widehat{E}$. Let $S=G^{-1} L^{-1} G(T)$. Then $S$ is maximally monotone and quasidense, and $S^{\mathbb{F}}=T$.

Proof. Fact 8.6 (with $E$ replaced by $E^{*}$ ) implies that $T$ is maximally monotone. The result now follows from Theorem 8.5(c).

Example 8.8. Let $E=c_{0}$, and define $T: E^{*}=\ell_{1} \rightarrow E^{* *}=\ell_{\infty}$ by $(T x)_{n}=\sum_{k \geq n} x_{k} . \quad T$ is the "tail operator". Let $S=G^{-1} L^{-1} G(T)$. It was proved in [18, Example 7.10, pp. 1034-1035] that $T$ is not quasidense. Thus, from Theorems 8.2 and $8.7, S$ is maximally monotone and quasidense, but $S^{\mathbb{F}}$ is maximally monotone and not quasidense. This example answers in the negative the question posed in [18, Problem 12.7, p. 1047] as to whether the Fitzpatrick extension of a quasidense maximally monotone multifunction is necessarily quasidense. $S$ can be represented in matrix form by

$$
\left(\begin{array}{c}
(S x)_{1} \\
(S x)_{2} \\
(S x)_{3} \\
(S x)_{4} \\
(S x)_{5} \\
\vdots
\end{array}\right)=\left(\begin{array}{cccccc}
1 & -1 & 0 & 0 & 0 & \ldots \\
0 & 1 & -1 & 0 & 0 & \ldots \\
0 & 0 & 1 & -1 & 0 & \ldots \\
0 & 0 & 0 & 1 & -1 & \ldots \\
0 & 0 & 0 & 0 & 1 & \ldots \\
\vdots & \vdots & \vdots & \vdots & \vdots & \ddots
\end{array}\right)\left(\begin{array}{c}
x_{1} \\
x_{2} \\
x_{3} \\
x_{4} \\
x_{5} \\
\vdots
\end{array}\right)
$$

and $D(S)=\left\{x \in c_{0}: \sum_{i=1}^{\infty}\left|x_{i}-x_{i+1}\right|<\infty\right\}$.

\section{SUM THEOREM WITH RANGE CONSTRAINTS}

Theorem 9.1 below has applications to the classification of maximally monotone multifunctions. See [19, Theorems 8.2 and 10.3].

Theorem 9.1 (Sum theorem with range constraints). Let $S, T: E \rightrightarrows E^{*}$ be closed, quasidense and monotone. Then $(\mathrm{a}) \Longrightarrow(\mathrm{b}) \Longrightarrow(\mathrm{c}) \Longrightarrow(\mathrm{d})$ :

(a) $R(S) \cap \operatorname{int} R(T) \neq \emptyset$ or int $R(S) \cap R(T) \neq \emptyset$.

(b) $\bigcup_{\lambda>0} \lambda[R(S)-R(T)]=E^{*}$.

(c) $\bigcup_{\lambda>0} \lambda\left[\pi_{2} \operatorname{dom} \varphi_{S}-\pi_{2} \operatorname{dom} \varphi_{T}\right]$ is a closed subspace of $E^{*}$.

(d) The multifunction $E \rightrightarrows E^{*}$ defined by $y \mapsto\left(S^{\mathbb{F}}+T^{\mathbb{F}}\right)^{-1}(\widehat{y})$ is closed, quasidense and monotone.

(e) If, further, $R\left(T^{\mathbb{F}}\right) \subset \widehat{E}$, then the parallel sum $\left(S^{-1}+T^{-1}\right)^{-1}$ is closed, monotone and quasidense.

Proof. It is immediate (using (7.1)) that $(\mathrm{a}) \Longrightarrow(\mathrm{b}) \Longrightarrow(\mathrm{c})$. Now suppose that (c) is satisfied. From Theorem 6.1, $S$ and $T$ are maximally monotone, and so (6.9) implies that $\varphi_{S}, \varphi_{T} \in \mathscr{P} \mathscr{C} \mathscr{L} \mathscr{S} \mathscr{C}\left(E \times E^{*}\right)$, $\varphi_{S}, \varphi_{T} \geq q_{L}$ on $E \times E^{*}$, coinc $\left[\varphi_{S}\right]=G(S)$ and coinc $\left[\varphi_{T}\right]=G(T)$, and we can apply Theorem 5.4 with 
$f:=\varphi_{S}$ and $g:=\varphi_{T}$. Thus $\left(\varphi_{S} \oplus_{1} \varphi_{T}\right)^{@} \geq q_{L}$ on $E \times E^{*}, \operatorname{coinc}\left[\left(\varphi_{S} \oplus_{1} \varphi_{T}\right)^{@}\right]$ is closed and quasidense, and $\left(y, y^{*}\right) \in \operatorname{coinc}\left[\left(\varphi_{S} \oplus_{1} \varphi_{T}\right)^{@}\right]$ if, and only if, there exist $u^{* *}, v^{* *} \in E^{* *}$ such that

$$
\left(y^{*}, u^{* *}\right) \in \operatorname{dcoinc}\left[\varphi_{S}^{*}\right],\left(y^{*}, v^{* *}\right) \in \operatorname{dcoinc}\left[\varphi_{T}^{*}\right] \text { and } u^{* *}+v^{* *}=\widehat{y} .
$$

From (8.1), this is equivalent to the statement: " $u^{* *} \in S^{\mathbb{F}}\left(y^{*}\right), v^{* *} \in T^{\mathbb{F}}\left(y^{*}\right)$ and $u^{* *}+v^{* *}=\widehat{y}$ ", that is to say, " $\widehat{y} \in\left(S^{\mathbb{F}}+T^{\mathbb{F}}\right)\left(y^{*}\right)$ ". This gives $(\mathrm{d})$.

(e) Now suppose that $R\left(T^{\mathbb{F}}\right) \subset \widehat{E}$ and $\left(y, y^{*}\right) \in \operatorname{coinc}\left[\left(\varphi_{S} \oplus_{1} \varphi_{T}\right)^{@}\right]$. Then the element $v^{* *}$ in (9.1) is actually in $\widehat{E}$, and so there exists $v \in E$ such that $\widehat{v}=v^{* *} \in T^{\mathbb{F}}\left(y^{*}\right)$. (8.3) now implies that $\left(v, y^{*}\right) \in G(T)$, that is to say $v \in T^{-1} y^{*}$. From (9.1) again, $u^{* *}=\widehat{y-v}$, and a repetition of the argument above gives $y-v \in S^{-1} y^{*}$. Consequently, we have $y=v+(y-v) \in\left(S^{-1}+T^{-1}\right) y^{*}$, that is to say $y^{*} \in\left(S^{-1}+T^{-1}\right)^{-1} y$. Thus we have proved that coinc $\left[\left(\varphi_{S} \oplus_{1} \varphi_{T}\right)^{\circledR}\right] \subset G\left(\left(S^{-1}+T^{-1}\right)^{-1}\right)$ On the other hand, from (8.3) and (9.1), we always have $G\left(\left(S^{-1}+T^{-1}\right)^{-1}\right) \subset \operatorname{coinc}\left[\left(\varphi_{S} \oplus_{1} \varphi_{T}\right)^{@}\right]$, completing the proof of (e).

\section{ANOTHER MAXIMALLY MONOTONE NON-QUASIDENSE MULTIFUNCTION}

In Bueno-Svaiter, [3, Proposition 1, pp. 84-85] an example is given of a maximally monotone skew linear operator from a subspace of $c_{0}$ into $\ell_{1}$ which is maximally monotone but not of type $(D)$, thus answering in the negative a conjecture of J. Borwein. As observed in [19, Remark 10.4, pp. 21-22], a maximally monotone multifunction is of type (D) if, and only if, it is quasidense, so the Bueno-Svaiter example provides a maximally monotone non-quasidense multifunction on $c_{0}$. In this section, we discuss a slight modification of this multifunction. Ironically, it is easier to establish the non-quasidensity than the maximal monotonicity.

Definition 10.1. If $\left(x_{n}\right)$ is a real sequence such that $\sum_{k=1}^{\infty} x_{k}$ is convergent, we define the tail sequence of $x,\left(t(x)_{n}\right)$, by, for all $n \geq 1, t(x)_{n}=\sum_{k=n}^{\infty} x_{k}$. Clearly

$$
t(x) \in c_{0} \quad \text { and, for all } j \geq 1, \quad x_{j}=t(x)_{j}-t(x)_{j+1} .
$$

Let

$$
K:=\left\{x=\left(x_{i}\right)_{i \geq 1}: \quad \sum_{i=1}^{\infty} x_{i}=0 \quad \text { and } \quad \sum_{p=1}^{\infty}\left|t(x)_{p}+t(x)_{p+1}\right|<\infty\right\} .
$$

$K$ is a vector subspace of $c_{0}$. Let $x \in K$. For all $j \geq 1$, let

$$
(S x)_{j}:=-t(x)_{j}-t(x)_{j+1} .
$$

Clearly, $S x \in \ell_{1} . S$ can be represented in matrix form by

$$
\left(\begin{array}{c}
(S x)_{1} \\
(S x)_{2} \\
(S x)_{3} \\
(S x)_{4} \\
(S x)_{5} \\
\vdots
\end{array}\right)=\left(\begin{array}{cccccc}
-1 & -2 & -2 & -2 & -2 & \ldots \\
0 & -1 & -2 & -2 & -2 & \ldots \\
0 & 0 & -1 & -2 & -2 & \ldots \\
0 & 0 & 0 & -1 & -2 & \ldots \\
0 & 0 & 0 & 0 & -1 & \ldots \\
\vdots & \vdots & \vdots & \vdots & \vdots & \ddots
\end{array}\right)\left(\begin{array}{c}
x_{1} \\
x_{2} \\
x_{3} \\
x_{4} \\
x_{5} \\
\vdots
\end{array}\right)
$$

If $x \in K$ then $t(x)_{1}=0$ and so, for all $k \geq 1$,

$$
\left.\begin{array}{rl}
\sum_{j=1}^{k} x_{j}(S x)_{j} & =\sum_{j=1}^{k}\left(t(x)_{j}-t(x)_{j+1}\right)\left(-t(x)_{j}-t(x)_{j+1}\right) \\
& =t(x)_{k+1}^{2}-t(x)_{1}^{2}=t(x)_{k+1}^{2} .
\end{array}\right\}
$$

Letting $k \rightarrow \infty$ in (10.4), for all $x \in K$,

$$
\langle x, S x\rangle=\lim _{k \rightarrow \infty} \sum_{j=1}^{k} x_{j}(S x)_{j}=\lim _{k \rightarrow \infty} t(x)_{k+1}^{2}=0 .
$$

If $x \in c_{0} \backslash K$, we define $S x:=\emptyset$. Thus $S: c_{0} \rightrightarrows \ell_{1}$ is at most single-valued, linear and skew and $D(S)=K$. If $i \geq 1$, write $e^{(i)}$ for the sequence $(0, \ldots, 0,1,0,0, \ldots)$, with the 1 in the $i$ th place. 
Lemma 10.2. Let $j \geq 1$. Then

$$
e^{(j)}-e^{(j+1)} \in K \quad \text { and } \quad S\left(e^{(j)}-e^{(j+1)}\right)=e^{(j)}+e^{(j+1)} .
$$

In other words,

$$
\left(e^{(j)}-e^{(j+1)}, e^{(j)}+e^{(j+1)}\right) \in G(S) .
$$

Proof. Let $x:=e^{(j)}-e^{(j+1)}$. It is easily seen that $t(x)=-e^{(j+1)}$. So, for all $p \geq 1$,

$$
(S x)_{p}=e_{p}^{(j+1)}+e_{p+1}^{(j+1)}= \begin{cases}0+0=0 & \text { if } p<j \\ 0+1=1 & \text { if } p=j \\ 1+0=1 & \text { if } p=j+1 \\ 0+0=0 & \text { if } p>j+1\end{cases}
$$

This gives the desired result. Alternatively, we can simply subtract the $(j+1)$ st column from the $j$ th column of the matrix in (10.3).

Theorem 10.3. $S$ is skew and maximally monotone but not quasidense.

Proof. From (10.5), $S$ is skew. Now let $\left(x, x^{*}\right) \in c_{0} \times \ell_{1}$ and,

$$
\text { for all } z \in K,\left\langle z-x, S z-x^{*}\right\rangle \geq 0 \text {. }
$$

From (10.5), $\langle z, S z\rangle=0$, and so (10.6) reduces to $\langle x, S z\rangle+\left\langle z, x^{*}\right\rangle \leq\left\langle x, x^{*}\right\rangle$. Since $K$ is a vector space, this implies that

$$
\left\langle x, x^{*}\right\rangle \geq 0 \text { and, for all } z \in K,\langle x, S z\rangle=-\left\langle z, x^{*}\right\rangle .
$$

Lemma 10.2 and (10.7) imply that, for all $j \geq 1$,

$$
x_{j}+x_{j+1}=\left\langle x, e^{(j)}+e^{(j+1)}\right\rangle=-\left\langle e^{(j)}-e^{(j+1)}, x^{*}\right\rangle=-x_{j}^{*}+x_{j+1}^{*} .
$$

Consequently, for all $n \geq 1$,

$$
x_{1}+x_{2}+\cdots+x_{2 n-1}+x_{2 n}=-x_{1}^{*}+x_{2}^{*}+\cdots-x_{2 n-1}^{*}+x_{2 n}^{*} .
$$

Adding $x_{2 n+1}$ to both sides of this equation,

$$
x_{1}+x_{2}+\cdots+x_{2 n-1}+x_{2 n}+x_{2 n+1}=-x_{1}^{*}+x_{2}^{*}+\cdots-x_{2 n-1}^{*}+x_{2 n}^{*}+x_{2 n+1} .
$$

Using the fact that $x \in c_{0}, x^{*} \in \ell_{1}$ and a simple interleaving argument, we see that $\sum_{i=1}^{\infty} x_{i}=\sum_{i=1}^{\infty}(-1)^{i} x_{i}^{*}$. Since we now know that $\sum_{i=1}^{\infty} x_{i}$ is convergent, we can use the notation of Definition 10.1. Thus

$$
t(x)_{1}=\sum_{i=1}^{\infty}(-1)^{i} x_{i}^{*} .
$$

Let $j \geq 1$. Using the same argument as above but starting the summation at $i=j$ instead of $i=1$, $t(x)_{j}=-x_{j}^{*}+x_{j+1}^{*}-+\cdots$. Replacing $j$ by $j+1, t(x)_{j+1}=-x_{j+1}^{*}+x_{j+2}^{*}-+\cdots$ and so, by addition, $x_{j}^{*}=-t(x)_{j}-t(x)_{j+1}$. From (10.2), $x_{j}^{*}=(S x)_{j}$. So $S x=x^{*} \in \ell_{1}$. Furthermore,

$$
\left\langle x, x^{*}\right\rangle=\sum_{j=1}^{\infty} x_{j} x_{j}^{*}=\sum_{j=1}^{\infty}\left(t(x)_{j}-t(x)_{j+1}\right)\left(-t(x)_{j}-t(x)_{j+1}\right)=-t(x)_{1}^{2},
$$

and (10.7) now gives $t(x)_{1}=0$. Thus, from (10.1), $x \in K$ and $\left(x, x^{*}\right) \in G(S)$. This completes the proof of the maximal monotonicity of $S$.

We now prove that $S$ is not quasidense. To this end, let $x \in K$. Then, from (10.2), $\sum_{j=1}^{\infty}(-1)^{j}(S x)_{j}=$ $\left(t(x)_{1}+t(x)_{2}\right)-\left(t(x)_{2}+t(x)_{3}\right)+\left(t(x)_{3}+t(x)_{4}\right) \cdots=t(x)_{1}=0$, thus

$$
(S x)_{1}=\sum_{j=2}^{\infty}(-1)^{j}(S x)_{j}, \text { from which }\left|(S x)_{1}\right| \leq \sum_{j=2}^{\infty}\left|(S x)_{j}\right| .
$$


Thus $2\left|(S x)_{1}\right| \leq \sum_{j=1}^{\infty}\left|(S x)_{j}\right|=\|S x\|_{1}$. Since $(S x)_{1}=-t(x)_{1}-t(x)_{2}=t(x)_{1}-t(x)_{2}=x_{1},\|S x\|_{1}^{2} \geq 4 x_{1}^{2}$. From (10.5), $\left\langle x-e^{(1)}, S x\right\rangle=\langle x, S x\rangle-(S x)_{1}=-x_{1}$, and so

$$
\begin{aligned}
r_{L}\left((x, S x)-\left(e^{(1)}, 0\right)\right) & =\frac{1}{2}\left\|x-e^{(1)}\right\|_{\infty}^{2}+\frac{1}{2}\|S x\|_{1}^{2}+\left\langle x-e^{(1)}, S x\right\rangle \\
& \geq \frac{1}{2}\left(x_{1}-1\right)^{2}+2 x_{1}^{2}-x_{1} \geq \frac{5}{2} x_{1}^{2}-2 x_{1}+\frac{1}{2} \\
& =\frac{5}{2}\left(x_{1}-\frac{2}{5}\right)^{2}+\frac{1}{10} \geq \frac{1}{10} .
\end{aligned}
$$

This completes the proof that $S$ is not quasidense.

Remark 10.4. As we observed above, $D(S)=K \neq c_{0}$. Also, the quasidense operator, $S: c_{0} \rightrightarrows \ell_{1}$ defined in Example 8.8 has $D(S) \neq c_{0}$. This leads to the following problem.

Problem 10.5. Is every maximally monotone multifunction $S: c_{0} \rightrightarrows \ell_{1}$ such that $D(S)=c_{0}$ quasidense?

It is natural to ask whether Theorem 8.5(b) can be used to establish the maximal monotonicity of $S$ in Theorem 10.3. Theorem 10.7 below shows that this is impossible. We now give a preliminary lemma:

Lemma 10.6. Let $S: c_{0} \rightrightarrows \ell_{1}$ be as in Definition 10.1 and Theorem 10.3, $\left(x, x^{*}\right) \in G(S)$ and $\omega^{* *}:=$ $(-1,1,-1,1,-1, \ldots) \in \ell_{\infty}$. Then $\left\langle x^{*}, \omega^{* *}\right\rangle=0$.

Proof. $\left(x, x^{*}\right) \in c_{0} \times \ell_{1}$ and, from (10.1) and (10.2), $\sum_{i=1}^{\infty} x_{i}=0$ and, for all $j \geq 1, x_{j}^{*}:=-t(x)_{j}-t(x)_{j+1}$. Thus, for all $j \geq 1$,

$$
-x_{j}^{*}+x_{j+1}^{*}=t(x)_{j}+t(x)_{j+1}-t(x)_{j+1}-t(x)_{j+2}=x_{j}+x_{j+1} .
$$

Consequently,

$$
\begin{aligned}
\left\langle x^{*}, \omega^{* *}\right\rangle & =\left(-x_{1}^{*}+x_{2}^{*}\right)+\left(-x_{3}^{*}+x_{4}^{*}\right)+\left(-x_{5}^{*}+x_{6}^{*}\right)+\ldots \\
& =\left(x_{1}+x_{2}\right)+\left(x_{3}+x_{4}\right)+\left(x_{5}+x_{6}\right)+\cdots=\sum_{i=1}^{\infty} x_{i}=0 .
\end{aligned}
$$

This gives the desired result.

Theorem 10.7. Let $S$ be as in Theorem 10.3 and Theorem 10.3, $T: \ell_{1} \rightrightarrows \ell_{\infty}, R(T) \subset \widehat{c_{0}}$ and $S=$ $G^{-1} L^{-1} G(T)$. Then $T$ is not maximally monotone.

Proof. Let $\left(y^{*}, y^{* *}\right) \in G(T)$. From the proof of Theorem 8.5(a), there exists $\left(y, y^{*}\right) \in G(S)$ such that $\widehat{y}=y^{* *}$, and Lemma 10.6 implies that $\left\langle y^{*}, \omega^{* *}\right\rangle=0$. From (10.5), $\left\langle y^{*}, y^{* *}\right\rangle=\left\langle y, y^{*}\right\rangle=\langle y, S y\rangle=0$, from which $\left\langle y^{*}-0, y^{* *}-\omega^{* *}\right\rangle=\left\langle y^{*}, y^{* *}\right\rangle-\left\langle y^{*}, \omega^{* *}\right\rangle=0$. Thus $\left(0, \omega^{* *}\right)$ is monotonically related to $G(T)$. However, $\omega^{* *} \notin \widehat{c_{0}} \supset R(T)$, and so $\left(0, \omega^{* *}\right) \notin G(T)$. This completes the proof of Theorem 10.7.

\section{The BUENO-SVAiter CONSTRUCTION}

In Example 8.8, we gave an example of a quasidense maximally monotone multifunction with a nonquasidense Fitzpatrick extension. In this section, we give a construction, due to Bueno and Svaiter, that produces another example of a similar phenonemon. Definition 11.1 is patterned after Bueno, [1, Theorem 2.7, pp. 13-14]. It would be interesting to find a scheme that includes both the example of Example 8.8, and also examples of the kind considered in this section.

Definition 11.1. Let $E$ be a Banach space and $e^{* *} \in E^{* *} \backslash \widehat{E}$. We define $k: E^{*} \rightarrow \mathbb{R}$ by $k\left(y^{*}\right)=\left\langle y^{*}, e^{* *}\right\rangle^{2}$. $k$ is a convex, continuous function on $E^{*}$. Let $T: E^{*} \rightarrow E^{* *}$ be a linear map and $R(T) \subset \widehat{E}$. Suppose that

$$
\text { for all } x^{*} \in E^{*},\left\langle x^{*}, T x^{*}\right\rangle=k\left(x^{*}\right) \geq 0 .
$$

In what follows, "lin" stands for "linear hull of".

Lemma 11.2. $\operatorname{dom} k^{*}=\operatorname{lin}\left\{e^{* *}\right\}$ and, for all $\mu \in \mathbb{R}, k^{*}\left(2 \mu e^{* *}\right)=\mu^{2}$. 
Proof. If $z^{* *} \notin \operatorname{lin}\left\{e^{* *}\right\}$ then, from a well known algebraic result, there exists $z^{*} \in E^{*}$ so that $\left\langle z^{*}, e^{* *}\right\rangle=0$ but $\left\langle z^{*}, z^{* *}\right\rangle \neq 0$. Thus, for all $\lambda \in \mathbb{R}, k^{*}\left(z^{* *}\right) \geq\left\langle\lambda z^{*}, z^{* *}\right\rangle-\left\langle\lambda z^{*}, e^{* *}\right\rangle^{2}=\lambda\left\langle z^{*}, z^{* *}\right\rangle$, and by taking $\lambda$ large and of the appropriate sign, $k^{*}\left(z^{* *}\right)=\infty$. Thus $\operatorname{dom} k^{*} \subset \operatorname{lin}\left\{e^{* *}\right\}$. If now $\mu \in \mathbb{R}$ then $k^{*}\left(2 \mu e^{* *}\right)=$ $\sup _{y^{*} \in E^{*}}\left[2 \mu\left\langle y^{*}, e^{* *}\right\rangle-\left\langle y^{*}, e^{* *}\right\rangle^{2}\right]$. Since $e^{* *} \neq 0$, as $y^{*}$ runs through $E^{*},\left\langle y^{*}, e^{* *}\right\rangle$ runs through $\mathbb{R}$, and so (by elementary calaculus or completing the square) $k^{*}\left(2 \mu e^{* *}\right)=\sup _{\lambda \in \mathbb{R}}\left[2 \mu \lambda-\lambda^{2}\right]=\mu^{2}$.

Theorem 11.3. $T$ is not quasidense.

Proof. We start off by proving that

$$
\text { If } z^{* * *} \in E^{* * *},\left\langle\widehat{E}, z^{* * *}\right\rangle=\{0\} \text { and } \lambda \in \mathbb{R} \text { then } \theta_{T}\left(e^{* *}, \lambda z^{* * *}\right)=\frac{1}{4} .
$$

To this end, let $z^{* * *}$ and $\lambda$ be as in (11.2). From (11.1) and the definition of $T$, for all $x^{*} \in E^{*},\left\langle x^{*}, T x^{*}\right\rangle=$ $k\left(x^{*}\right)$, and (6.4) and Lemma 11.2 give

$$
\begin{aligned}
\theta_{T}\left(e^{* *}, \lambda z^{* * *}\right) & =\sup _{x^{*} \in E^{*}}\left[\left\langle x^{*}, e^{* *}\right\rangle+\lambda\left\langle T x^{*}, z^{* * *}\right\rangle-\left\langle x^{*}, T x^{*}\right\rangle\right] \\
& =\sup _{x^{*} \in E^{*}}\left[\left\langle x^{*}, e^{* *}\right\rangle+0-k\left(x^{*}\right)\right]=k^{*}\left(e^{* *}\right)=\frac{1}{4} .
\end{aligned}
$$

This completes the proof of (11.2). If $T$ were quasidense then, from (11.2) and Corollary 3.9, if $z^{* * *} \in E^{* * *}$ and $\left\langle\widehat{E}, z^{* * *}\right\rangle=\{0\}$ then, for all $\lambda \in \mathbb{R}$,

$$
\frac{1}{4}=\theta_{T}\left(e^{* *}, \lambda z^{* * *}\right) \geq\left\langle e^{* *}, \lambda z^{* * *}\right\rangle=\lambda\left\langle e^{* *}, z^{* * *}\right\rangle .
$$

Letting $\lambda \rightarrow \pm \infty,\left\langle e^{* *}, z^{* * *}\right\rangle=0$. So we would have $\left\langle e^{* *}, z^{* * *}\right\rangle=0$ whenever $\left\langle\widehat{E}, z^{* * *}\right\rangle=\{0\}$. Since $\widehat{E}$ is a closed subspace of $E^{* *}$, it would follow that $e^{* *} \in \widehat{E}$, violating the assumption in Definition 11.1.

Theorem 11.4. Let $S=G^{-1} L^{-1} G(T)$ (see Theorem 8.5). Then:

(a) $S$ is maximally monotone and quasidense, and $S^{\mathbb{F}}=T$.

(b) $S^{\mathbb{F}}$ is maximally monotone but not quasidense.

Proof. (a) is immediate from Definition 11.1 and Theorem 8.7, and (b) is immediate from Theorem 8.2, (a) and Theorem 11.3.

For the rest of this section, we shall consider some of the more technical properties of $\theta_{S}$, with $S=$ $G^{-1} L^{-1} G(T)$ as in Theorems 8.5 and 11.4. We now give a preliminary lemma:

Lemma 11.5. For all $x^{*}, y^{*} \in E^{*},\left\langle y^{*}, T x^{*}\right\rangle=\left\langle x^{*}, 2\left\langle y^{*}, e^{* *}\right\rangle e^{* *}-T y^{*}\right\rangle$.

Proof. We have

$$
\begin{aligned}
\left\langle y^{*}, T x^{*}\right\rangle+\left\langle x^{*}, T y^{*}\right\rangle & =\frac{1}{2}\left\langle x^{*}+y^{*}, T x^{*}+T y^{*}\right\rangle-\frac{1}{2}\left\langle x^{*}-y^{*}, T x^{*}-T y^{*}\right\rangle \\
& =\frac{1}{2} k\left(x^{*}+y^{*}\right)-\frac{1}{2} k\left(x^{*}-y^{*}\right)=2\left\langle x^{*}, e^{* *}\right\rangle\left\langle y^{*}, e^{* *}\right\rangle .
\end{aligned}
$$

The result follows easily from this.

Theorem 11.6. Let $\left(y^{*}, y^{* *}\right) \in E^{*} \times E^{* *}$. Then

$$
\left(y^{*}, y^{* *}\right) \in \operatorname{dom} \theta_{S} \Longleftrightarrow 2\left\langle y^{*}, e^{* *}\right\rangle e^{* *}-T y^{*}+y^{* *} \in \operatorname{lin}\left\{e^{* *}\right\} .
$$

It follows that $\operatorname{dom} \theta_{S}$ is a linear subpace of $E^{*} \times E^{* *}$. Furthermore, for all $\left(y^{*}, y^{* *}\right) \in \operatorname{dom} \theta_{S}$, there exists a unique value of $\mu \in \mathbb{R}$ such that

$$
2\left\langle y^{*}, e^{* *}\right\rangle e^{* *}-T y^{*}+y^{* *}=2 \mu e^{* *} \text {, and then } \theta_{S}\left(y^{*}, y^{* *}\right)=\mu^{2} .
$$

Proof. It follows from (6.4) and (11.1) that

$$
\theta_{S}\left(y^{*}, y^{* *}\right)=\sup _{x^{*} \in E^{*}}\left[\left\langle y^{*}, T x^{*}\right\rangle+\left\langle x^{*}, y^{* *}\right\rangle-k\left(x^{*}\right)\right] .
$$


Thus, from Lemma 11.5,

$$
\begin{aligned}
\theta_{S}\left(y^{*}, y^{* *}\right) & =\sup _{x^{*} \in E^{*}}\left[\left\langle x^{*}, 2\left\langle y^{*}, e^{* *}\right\rangle e^{* *}-T y^{*}+y^{* *}\right\rangle-k\left(x^{*}\right)\right] \\
& =k^{*}\left(2\left\langle y^{*}, e^{* *}\right\rangle e^{* *}-T y^{*}+y^{* *}\right) .
\end{aligned}
$$

(11.3) now follows from Lemma 11.2. Since $e^{* *} \neq 0$, for all $\left(y^{*}, y^{* *}\right) \in \operatorname{dom} \theta_{S}$ there exists a unique $\mu \in \mathbb{R}$ such that $2\left\langle y^{*}, e^{* *}\right\rangle e^{* *}-T y^{*}+y^{* *}=2 \mu e^{* *}$, and the rest of (11.4) follows from another application of Lemma 11.2.

Corollary 11.7. $\operatorname{dom} \varphi_{S}=G(S)$ and $\theta_{S}=\varphi_{S}^{*}$ on $E^{*} \times E^{* *}$.

Proof. Let $\left(x, x^{*}\right) \in \operatorname{dom} \varphi_{S}$. From (6.5), $\left(x^{*}, \widehat{x}\right) \in \operatorname{dom} \theta_{S}$. Theorem 11.6 now gives a unique value of $\mu \in \mathbb{R}$ such that $2\left\langle x^{*}, e^{* *}\right\rangle e^{* *}-T x^{*}+\widehat{x}=2 \mu e^{* *}$. Thus $\widehat{E} \ni \widehat{x}-T x^{*}=2\left(\mu-\left\langle x^{*}, e^{* *}\right\rangle\right) e^{* *}$. From Definition 11.1, $e^{* *} \notin \widehat{E}$, and so $\mu-\left\langle x^{*}, e^{* *}\right\rangle=0$, from which $\widehat{x}-T x^{*}=0$. It follows that $\left(x, x^{*}\right) \in G(S)$. Thus $\operatorname{dom} \varphi_{S} \subset G(S)$. The result now follows from Lemma 6.11 .

Since $S$ is quasidense, it follows from Theorem 6.15 that

$$
\operatorname{dcoinc}\left[\theta_{S}\right]=\operatorname{dcoinc}\left[\varphi_{S}{ }^{*}\right]=\operatorname{dcoinc}\left[\theta_{S}{ }^{@}\right] .
$$

Of course, we know the first equality in (11.5) from Corollary 11.7. The second equality in (11.5) leads naturally to the conjecture that $\theta_{S}{ }^{@}=\varphi_{S}{ }^{*}$ on $E^{*} \times E^{* *}$. As we show in Theorem 11.8 below, this conjecture fails in a spectacular way. This raises the question of finding the exact value of $\operatorname{dom} \theta_{S}{ }^{\circledR}$.

Theorem 11.8. Since $e^{* *} \neq 0$, there exists $y^{*} \in E^{*}$ so that $\left\langle y^{*}, e^{* *}\right\rangle=1$. Define $y^{* *} \in E^{* *}$ by $y^{* *}:=T y^{*}-2 e^{* *}$. Let $\lambda \in \mathbb{R}$. Then

$$
\theta_{S}\left(\lambda y^{*}, \lambda y^{* *}\right)=0 \text {, in particular, } \varphi_{S}^{*}\left(y^{*}, y^{* *}\right)=\theta_{S}\left(y^{*}, y^{* *}\right)=0 .
$$

but

$$
\theta_{S}{ }^{@}\left(y^{*}, y^{* *}\right)=\infty .
$$

Proof. We note that $2\left\langle\lambda y^{*}, e^{* *}\right\rangle e^{* *}-T \lambda y^{*}+\lambda y^{* *}=\lambda\left(2 e^{* *}-T y^{*}+y^{* *}\right)=0$, so (11.6) follows from (11.4). Let $\lambda<0$. From (4.9) and (11.6),

$$
\begin{aligned}
\theta_{S}{ }^{@}\left(y^{*}, y^{* *}\right) & =\sup _{\left(x^{*}, x^{* *}\right) \in E^{*} \times E^{* *}}\left[\left\langle\left(x^{*}, x^{* *}\right),\left(y^{* *}, \widehat{y^{*}}\right)\right\rangle-\theta_{S}\left(x^{*}, x^{* *}\right)\right] \\
& \geq\left\langle\lambda y^{*}, y^{* *}\right\rangle+\left\langle y^{*}, \lambda y^{* *}\right\rangle-\theta_{S}\left(\lambda y^{*}, \lambda y^{* *}\right)=2 \lambda\left\langle y^{*}, y^{* *}\right\rangle .
\end{aligned}
$$

However, $\left\langle y^{*}, y^{* *}\right\rangle=\left\langle y^{*}, T y^{*}-2 e^{* *}\right\rangle=\left\langle y^{*}, T y^{*}\right\rangle-2\left\langle y^{*}, e^{* *}\right\rangle$. It now follows from (11.1) that $\left\langle y^{*}, y^{* *}\right\rangle=\left\langle y^{*}, e^{* *}\right\rangle^{2}-2\left\langle y^{*}, e^{* *}\right\rangle=1-2=-1$, and so $\theta_{S}{ }^{@}\left(y^{*}, y^{* *}\right) \geq-2 \lambda$, and we obtain (11.7) by letting $\lambda \rightarrow-\infty$.

\section{A SPECIFIC NON-QUASIDENSE FITZPATRICK EXTENSION}

If $x^{*} \in \ell_{1}$ and $j \geq 1$, let $\tau_{j}:=\sum_{i=j}^{\infty} x_{i}^{*}$. Define the linear map $T: \ell_{1} \rightarrow \ell_{\infty}$ by

$$
\text { for all } j \geq 1,\left(T x^{*}\right)_{j}=\tau_{j}+\tau_{j+1} \text {. }
$$

Clearly $R(T) \subset \widehat{c_{0}}$. Let $e^{* *}:=(1,1,1,1, \ldots) \in \ell_{\infty} \backslash \widehat{c_{0}}$.

Remark 12.1. $T$ can be represented by

$$
\left(\begin{array}{c}
\left(T x^{*}\right)_{1} \\
\left(T x^{*}\right)_{2} \\
\left(T x^{*}\right)_{3} \\
\left(T x^{*}\right)_{4} \\
\left(T x^{*}\right)_{5} \\
\vdots
\end{array}\right)=\left(\begin{array}{cccccc}
1 & 2 & 2 & 2 & 2 & \ldots \\
0 & 1 & 2 & 2 & 2 & \ldots \\
0 & 0 & 1 & 2 & 2 & \ldots \\
0 & 0 & 0 & 1 & 2 & \ldots \\
0 & 0 & 0 & 0 & 1 & \ldots \\
\vdots & \vdots & \vdots & \vdots & \vdots & \ddots
\end{array}\right)\left(\begin{array}{c}
x_{1}^{*} \\
x_{2}^{*} \\
x_{3}^{*} \\
x_{4}^{*} \\
x_{5}^{*} \\
\vdots
\end{array}\right)
$$


Lemma 12.2. For all $x^{*} \in \ell_{1},\left\langle x^{*}, T x^{*}\right\rangle=\left\langle x^{*}, e^{* *}\right\rangle^{2} \geq 0$.

Proof. Let $j \geq 1$. Then $x_{j}^{*}\left(T x^{*}\right)_{j}=\left(\tau_{j}+\tau_{j+1}\right)\left(\tau_{j}-\tau_{j+1}\right)=\tau_{j}^{2}-\tau_{j+1}^{2}$. Since $x^{*} \in \ell_{1}, \lim _{k \rightarrow \infty} \tau_{k}=0$. Thus

$$
\sum_{j=1}^{\infty} x_{j}^{*}\left(T x^{*}\right)_{j}=\lim _{k \rightarrow \infty} \sum_{j=1}^{k} x_{j}^{*}\left(T x^{*}\right)_{j}=\lim _{k \rightarrow \infty} \sum_{j=1}^{k}\left(\tau_{j}^{2}-\tau_{j+1}^{2}\right)=\tau_{1}^{2},
$$

as required.

Theorem 12.3. Let $S=G^{-1} L^{-1} G(T)$. Then $S$ is maximally monotone and quasidense, and $S^{\mathbb{F}}=T$ is maximally monotone but not quasidense.

Proof. This is immediate from Lemma 12.2 and Theorem 11.4.

Remark 12.4. In this case we can give a direct proof that $T$ is not quasidense. For all $x^{*} \in \ell_{1}, T x^{*} \in \widehat{c_{0}}$ and so $\left\|T x^{*}-e^{* *}\right\|_{\infty} \geq 1$, and $\left\langle x^{*}, T x^{*}-e^{* *}\right\rangle=\left\langle x^{*}, T x^{*}\right\rangle-\left\langle x^{*}, e^{* *}\right\rangle=\left\langle x^{*}, e^{* *}\right\rangle^{2}-\left\langle x^{*}, e^{* *}\right\rangle$. Thus

$$
\begin{aligned}
r_{L}\left(\left(x^{*}, T x^{*}\right)-\left(0, e^{* *}\right)\right) & =\frac{1}{2}\left\|x^{*}\right\|_{1}^{2}+\frac{1}{2}\left\|T x^{*}-e^{* *}\right\|_{\infty}^{2}+\left\langle x^{*}, T x^{*}-e^{* *}\right\rangle \\
& \geq 0+\frac{1}{2}+\left\langle x^{*}, e^{* *}\right\rangle^{2}-\left\langle x^{*}, e^{* *}\right\rangle \\
& =\frac{1}{4}+\frac{1}{4}\left(2\left\langle x^{*}, e^{* *}\right\rangle-1\right)^{2} \geq \frac{1}{4} .
\end{aligned}
$$

Thus $T$ is not quasidense.

Remark 12.5. Let $x \in c_{0}$ and $x^{*} \in \ell_{1}$. Note that $\tau \in c_{0}$. If $\left(x, x^{*}\right) \in G(S)$ then, for all $j \geq 1, x_{j}=$ $\tau_{j}+\tau_{j+1}$. It follows that $x_{j}-x_{j+1}=\tau_{j}-\tau_{j+2}=x_{j}^{*}+x_{j+1}^{*}$. Thus $x_{j}^{*}=\left(x_{j}^{*}+x_{j+1}^{*}\right)-\left(x_{j+1}^{*}+x_{j+2}^{*}\right) \pm \ldots$ $=\left(x_{j}-x_{j+1}\right)-\left(x_{j+1}-x_{j+2}\right) \pm \ldots$. It follows that $S$ can be represented in matrix form on the appropriate domain by

$$
\left(\begin{array}{c}
(S x)_{1} \\
(S x)_{2} \\
(S x)_{3} \\
(S x)_{4} \\
(S x)_{5} \\
\vdots
\end{array}\right)=\left(\begin{array}{cccccc}
1 & -2 & 2 & -2 & 2 & \ldots \\
0 & 1 & -2 & 2 & -2 & \ldots \\
0 & 0 & 1 & -2 & 2 & \ldots \\
0 & 0 & 0 & 1 & -2 & \ldots \\
0 & 0 & 0 & 0 & 1 & \ldots \\
\vdots & \vdots & \vdots & \vdots & \vdots & \ddots
\end{array}\right)\left(\begin{array}{c}
x_{1} \\
x_{2} \\
x_{3} \\
x_{4} \\
x_{5} \\
\vdots
\end{array}\right)
$$

\section{Acknowledgement}

The author is grateful to the reviewer for a very careful reading of this paper.

\section{REFERENCES}

[1] O. Bueno, On non-type (D) operators in non-reflexive Banach spaces and closures of monotone operators in topological vector spaces, Thesis, IMPA, 2012.

[2] O. Bueno, B. F. Svaiter, A maximal monotone operator of type (D) for which maximal monotone extension to the bidual is not of type (D), J. Convex Anal. 19 (2012), 295-300.

[3] O. Bueno, B. F. Svaiter, A non-type (D) operator in $c_{0}$, Math. Program. Ser. B 139 (2013), 81-88.

[4] R.S. Burachik, B.F. Svaiter, Maximal monotonicity, conjugation and the duality product, Proc. Amer. Math. Soc. 131 (2003), 2379-2383.

[5] S. Fitzpatrick, Representing monotone operators by convex functions, Workshop/ Miniconference on Functional Analysis and Optimization (Canberra, 1988), 59-65, Proc. Centre Math. Anal. Austral. Nat. Univ., 20, Austral. Nat. Univ., Canberra, 1988.

[6] S.P. Fitzpatrick, R.R. Phelps, Some properties of maximal monotone operators on nonreflexive Banach spaces, Set-Valued Anal. 3 (1995), 51-69.

[7] J.-P. Gossez, Opérateurs monotones non linéaires dans les espaces de Banach non réflexifs, J. Math. Anal. Appl. 34 (1971), 371-395. 
[8] H. König, Some Basic Theorems in Convex Analysis, in “Optimization and operations research", edited by B. Korte, North-Holland, 1982.

[9] N. Krylov, Properties of monotone mappings, Lith. Math. J. 22 (1982), 140-145.

[10] J.-E. Martínez-Legaz, M. Théra, A convex representation of maximal monotone operators, J. Nonlinear Convex Anal. 2 (2001), 243-247.

[11] J.-P. Penot, The relevance of convex analysis for the study of monotonicity, Nonlinear Anal. 58 (2004), 855871.

[12] R.R. Phelps, S. Simons, Unbounded linear monotone operators on nonreflexive Banach spaces, J. Convex Analysis 5 (1998), 303-328.

[13] R.T. Rockafellar, Extension of Fenchel's duality theorem for convex functions, Duke Math. J. 33 (1966), 81-89.

[14] S. Simons, The range of a monotone operator, J. Math. Anal. Appl. 199 (1996), 176-201.

[15] S. Simons, From Hahn-Banach to monotonicity, Lecture Notes in Mathematics, vol. 1693, second edition, Springer-Verlag, 2008.

[16] S. Simons, Quadrivariate existence theorems and strong representability, Optimization 60 (2011), 875-891.

[17] S. Simons, The Asymmetric sandwich theorem, J. Convex Anal. 20 (2013), 107-124.

[18] S. Simons, "Densities" and maximal monotonicity, J. Convex Anal. 23 (2016), 1017-1050.

[19] S. Simons, Quasidense monotone multifunctions, Set-Valued Var. Anal. 26 (2018), 5-26.

[20] S. Simons, X. Wang, Ubiquitous subdifferentials, $r_{L}$-density and maximal monotonicity, Set-Valued Var. Anal. 23 (2015), 631-642.

[21] S. Simons, C. Zălinescu, Fenchel duality, Fitzpatrick functions and maximal monotonicity, J. Nonlinear Convex Anal. 6 (2005), 1-22.

[22] M. D. Voisei, C. Zălinescu, Strongly-representable operators, J. Convex Anal. 16 (2009), 1011-1033.

[23] C. Zălinescu, Convex analysis in general vector spaces, World Scientific, 2002. https://doi.org/10.1142/5021 